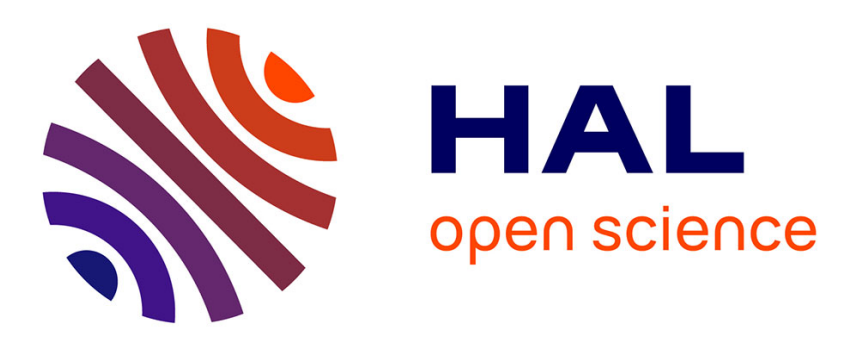

\title{
Intermittency of vertical density gradients at finescale and link with mixing processes
}

\author{
Pascale Bouruet-Aubertot, Joël Sommeria, Bernard Le Cann, Christophe \\ Koudella
}

\section{- To cite this version:}

Pascale Bouruet-Aubertot, Joël Sommeria, Bernard Le Cann, Christophe Koudella. Intermittency of vertical density gradients at finescale and link with mixing processes. Deep Sea Research Part II: Topical Studies in Oceanography, 2004, 51 (25-26), pp.2919-2941. 10.1016/j.dsr2.2004.09.007 . hal-00260759

\section{HAL Id: hal-00260759 \\ https://hal.science/hal-00260759}

Submitted on 10 Feb 2020

HAL is a multi-disciplinary open access archive for the deposit and dissemination of scientific research documents, whether they are published or not. The documents may come from teaching and research institutions in France or abroad, or from public or private research centers.
L'archive ouverte pluridisciplinaire HAL, est destinée au dépôt et à la diffusion de documents scientifiques de niveau recherche, publiés ou non, émanant des établissements d'enseignement et de recherche français ou étrangers, des laboratoires publics ou privés. 


\title{
Intermittency of vertical density gradients at finescale and link with mixing processes
}

\author{
Pascale Bouruet-Aubertot ${ }^{\mathrm{a}, *}$, J. Sommeria ${ }^{\mathrm{b}}$, B. Le Cann ${ }^{\mathrm{c}}$, C.R. Koudella ${ }^{\mathrm{d}}$ \\ ${ }^{\mathrm{a}}$ LODYC/IPSL, Université Pierre et Marie Curie/UMR 7617, Paris, France \\ ${ }^{\mathrm{b}}$ LEGI, Université Joseph Fourier, Grenoble, France \\ ${ }^{\mathrm{c}}$ LPO, Université de Bretagne occidentale, Brest, France \\ ${ }^{\mathrm{d}}$ DEAS, Harvard University, Cambridge, USA
}

Nonlinear interactions among internal gravity waves lead to intermittent breaking events with density overturning and vertical turbulent mixing. We analyze the statistics of density fluctuations generated in this process, using direct numerical simulations. We then check the relevance of these results for in situ density profiles measured during the ARCANE experiment on the continental slope west of the Iberian peninsula, a region with strong internal tides. We focus the analysis in the depth range [400-1000 $\mathrm{m}$ ] for which the temperature is uniform, and the density stratification is due mainly to salinity.

We verify in the numerical simulations that the histograms of vertical density gradients become strongly skewed at small vertical scales. This intermittency of density fluctuations can be attributed to the formation of sheets with strong stable density gradients. These sheets result from convergent motion produced by surrounding breaking events. We check that the vertical density gradients at finescale can be represented by a model of layers and sheets distributed with a Poisson statistics, with a mean number of sheets per unit length, $\mu$. We next show that the mean sheet spacing $\mu^{-1}$ is proportional to the Ozmidov scale (proportional to the square root of the energy dissipation). Using the classical link between energy dissipation and vertical eddy diffusivity $K_{\mathrm{d}}$, we deduce a link between the statistics of vertical density gradients and the vertical mixing, namely $K_{\mathrm{d}}$ is proportional to $N \mu^{-2}$.

For in situ measurements, we find a similar statistics of vertical density gradients, confirming earlier results obtained by Hayes et al. [J. Geophys. Res. 80 (3) 314] and Pinkel and Anderson [J. Phys. Oceanogr. 22 (1992) 773] in the open ocean thermocline. This allows us to estimate a rate of total energ y dissipation $2.4 \times 10^{-8} \mathrm{~W} / \mathrm{kg}$ and a vertical eddy diffusivity $5 \times 10^{-4} \mathrm{~m}^{2} \mathrm{~s}^{-1}$. Comparisons with other estimates of energy dissipation or vertical mixing would be required to validate this method.

\footnotetext{
*Corresponding author.

E-mail address: pba@lodyc.jussieu.fr (P. Bouruet-Aubertot).
} 


\section{Introduction}

Wavebreaking corresponds to the onset of turbulence, with irreversible energy dissipation, from an initially smooth wave field. It controls mixing in stably stratified fluids, as other sources of turbulence tend to be suppressed. In many parts of the deep ocean, a quasi-permanent level of random internal wave activity is maintained by various sources. Internal waves are in particular produced from baroclinic tides, themselves generated by the interaction of the main (barotropic) tides with the bottom topography. They are also produced by atmospheric forcing, especially during storms or cooling events. Steepening by wave-wave interactions and refraction by density fluctuations produces a cascade of wave energy to smaller scales with stronger nonlinearity until breaking occurs. Turbulence is thus sporadically produced, leading to vertical mixing of heat and solutant.

Note that internal waves are also important in the atmosphere. They are emitted by the effect of topography or storms and are amplified by air rarefaction as they propagate upward until breaking occurs. This has a significant influence on the vertical wind profile, as momentum from the lower atmosphere is transferred to the altitude of breaking. The resulting regimes of wave induced turbulence have common properties in the atmosphere and oceans.

These properties have been reproduced to some extent in laboratory experiments by Bénielli and Sommeria (1998) and in numerical simulations by Bouruet-Aubertot et al. (1996) and Carnevale et al. (2001). In these studies the internal waves are produced in a uniformly stratified fluid with no Coriolis effects. Furthermore, turbulence is induced by the breaking of a simple wave which is initially introduced, or permanently forced at large scale. These studies had primarily focused on spectral analysis and global energy dissipation and mixing effects. The goal of the present paper is twofold. The first one is to characterize the intermittency of the vertical density structure in such well controlled configurations and to relate these processes with mixing. The second is to apply these results to oceanic data and to provide an indirect estimate of mixing.
The wave induced turbulence is characterized by an energy spectrum scaling as $k_{z}^{-3}$ along the vertical as observed both in the atmosphere (e.g. Alisse and Sidi, 2000), and in the ocean (e.g. Gargett et al., 1981). It connects the weakly nonlinear waves at the larger scales to the fully turbulent but sporadic micro-scales. This spectral domain is, therefore, important in determining the mixing properties of the internal wave field. It is called the buoyancy sub-range in the atmosphere (ranging from $100 \mathrm{~m}$ to a few $\mathrm{km}$ in vertical scales). In the oceans it corresponds to the so-called fine structure, or compliant waves (for vertical scales ranging from 1 to $10 \mathrm{~m}$ ). Such a $k_{z}^{-3}$ spectral range has been reproduced in the laboratory experiments of Bénielli and Sommeria (1998) and in the numerical simulations of (Bouruet-Aubertot et al., 1996; Carnevale et al., 2001).

Intermittency is known to be important in this wave induced turbulence. Sporadic occurrence of well mixed zones or strongly stratified layers have been documented. Baker and Gibson (1987) pointed out the requirement to take into account such intermittency when analyzing oceanic data of energy dissipation and mixing. However, the question of lognormality of dissipation rates in the ocean should be examined with care as underlined by Yamazaki and Lueck (1990) who proposed a robust method of computation of these quantities.

For weakly interacting internal wave fields we expect Gaussian statistics for both velocity and buoyancy. This results from general arguments of random phase (e.g. Olbers, 1976). The statistics then becomes more and more intermittent at smaller and smaller wavelengths, until the scale of the sporadic breaking events. This has been observed in several oceanic campaigns (Hayes et al., 1975; Desaubies and Gregg, 1981; Pinkel and Anderson, 1992), and recently confirmed in direct numerical simulations (Bouruet-Aubertot et al., 2004): while Gaussian distributions are obtained for buoyancy and velocity fluctuations at scales corresponding to resonantly interacting waves, skewed distributions are obtained for density fluctuations in the $k_{z}^{-3}$ range, becoming more and more skewed at smaller scales, corresponding to the rare occurrence of stratification steps. The energy 
dissipation is also intermittent: Gregg et al. (1986, 1993) found a lognormal distribution for the dissipation rate in the ocean (however perturbed by instrumental noise and undersampling).

Theoretical models have been developed in order to explain this strong skewness of the density structure. However, the link between energy dissipation (related to vertical mixing) and density statistics remains unclear.

Desaubies and Gregg (1981) have shown that the background temperature gradient is locally increased or decreased in an asymmetric way by the transport of a Gaussian wave field without energy dissipation. Their model provides an adequate description of oceanic observations for vertical scales larger than $2 \mathrm{~m}$. However it fails to describe smaller scales, at which breaking events and mixing occur.

An opposite model has been proposed by Hayes et al. (1975), which assumes that turbulent mixing cuts random steps in the initial uniform vertical stratification: each uniform layer is topped by a discontinuity sheet to keep up with the background gradient. The step edges are assumed randomly located, which results in Poisson statistics. Hayes et al. (1975) found a reasonable agreement with their oceanic data in the relevant range of vertical scales (1-10) m. Evidence for such stepwise structures has also been given in the atmosphere (Dalaudier et al., 1994).

Interestingly this vertical density structure has been retrieved from numerical simulations of internal gravity wavebreaking (Bouruet-Aubertot et al., 2004), opening the opportunity to test accurately the range of validity of these two models. It was found that the strain model is adequate for scales larger than 30 times the Ozmidov length scale, while the Poisson statistics provides a fairly good description for smaller scales. This should be viewed as a convenient practical description, although the underlying step and layer model is not realistic. Pinkel and Anderson (1992) have derived for instance the same Poisson statistics from an alternative physical model (a pile of layers with random density gradients). Taking into account strain variability as a function of vertical separation they later derived a model for the pdf of the
Richardson number (Pinkel and Anderson, 1997a) and then inferred an eddy diffusivity (Pinkel and Anderson, 1997b).

The key question addressed in this paper is to relate this statistics of density to mixing processes. To this aim we perform numerical computations that start with a simple initial condition (a largescale internal gravity wave). In this way, turbulence is produced by an intrinsic sequence of instabilities without external noise. A preliminary analysis of in situ oceanic measurements checking the relevance of the numerical results is also presented.

The paper is organized as follows: in Section 2 we describe the numerical model and introduce the physical parameters. In Section 3 we introduce the main characteristics of the wave induced turbulence and the relevant turbulent length scales. We next give evidence of the intermittency of the vertical density structure at small scales and characterize the most intense events that take the form of strongly stratified sheets in Section 4. In Section 5 we show how mixing can be inferred from the statistics of these stratified sheets. We finally analyze salinity and temperature profiles taken during the ARCANE experiments in Section 6.

\section{Numerical model}

We solve the Navier-Stokes equations in the Boussinesq approximation, either in the vertical plane (2D computations), or in three dimensions (3D computations). The domain is accordingly a vertical square or a cube. We use Cartesian coordinates $(x, z)$ or $(x, y, z)$, with the vertical coordinate $z$ directed upwards. The basic density profile, $\bar{\rho}$, is linearly decreasing with the vertical coordinate $z$ and the buoyancy frequency

$N^{2}=-\frac{g}{\rho_{0}} \frac{\mathrm{d} \bar{\rho}}{\mathrm{d} z}$,

is thus constant. In the Boussinesq approximation, the density variations are assumed to be small with respect to the mean density $\rho_{0}$.

In two dimensions, the condition of incompressibility is satisfied by introducing a stream 
function $\Psi$, related to the two velocity components $u$ and $w$ by $u=\partial_{z} \Psi, w=-\partial_{x} \Psi$. We solve the Boussinesq equations in terms of the vorticity $-\Delta \Psi$ and the buoyancy fluctuations $\rho^{*}$ : the actual density is therefore $\rho=\rho_{0}+\left(\rho_{0} / g\right) \rho^{*}$.

$$
\begin{aligned}
& \frac{\partial \Delta \Psi}{\partial t}+J(\Delta \Psi, \Psi)=\frac{\partial \rho^{*}}{\partial x}+v \nabla^{2} \Psi, \\
& \frac{\partial \rho^{*}}{\partial t}+J\left(\rho^{*}, \Psi\right)=-N^{2} \frac{\partial \Psi}{\partial x}+\kappa \nabla^{2} \rho^{*},
\end{aligned}
$$

where the non linear advective terms are written by means of the Jacobian $J$ and $v$ is the viscosity. The density is assumed to be a linear function of the transported quantity, temperature or salinity, with diffusivity $\kappa$, the Prandtl number $\operatorname{Pr}=(v / \kappa)$ is here taken equal to unity. We study standing waves in a square of side $\pi$ and we use periodic boundary conditions with symmetry (e.g. Canuto et al., 1988). The normal velocities at the boundaries are set to zero as well as density fluctuations at the horizontal boundaries, $z=0$ and $\pi$.

In three dimensions, we solve the equations of motion, with the Boussinesq approximation:

$$
\frac{\partial \vec{v}}{\partial t}+\vec{v} \cdot \nabla \vec{v}=-\frac{\vec{\nabla} p^{\prime}}{\rho_{0}}+\frac{\rho^{\prime}}{\rho_{0}} \vec{g}+v \nabla^{2} \vec{v},
$$

$\nabla \cdot \vec{v}=0$,

$$
\frac{\partial \rho^{\prime}}{\partial t}+\vec{v} \cdot \nabla \rho^{\prime}=\frac{\rho_{0}}{g} N^{2} w+\kappa \nabla^{2} \rho^{\prime},
$$

where $p^{\prime}$ is the pressure fluctuation divided by $\rho_{0}$ and $\vec{i}_{z}$ is the unit vector along the vertical direction oriented upward. As in the 2D case, we only consider standing waves with different amplitudes.

The parameters of the runs are given in Table 1. We analyzed mainly two cases. The first one is that of two-dimensional simulations with resolution $512^{2}$ and the second one is that of three-dimensional simulations, with lower resolution $128^{3}$, of the same wave with a perturbation superimposed.

The results of our calculations can be easily transposed to oceanic and atmospheric flows having the same Froude number and Reynolds number as those of our calculations. We look here at the evolution of a large-scale primary wave, of wavelength equal to $\lambda=2 \pi$ in the $x$ and $y$ directions, and which is evolving in a background
Table 1

Physical and numerical parameters of the simulations: $F r=$ $k_{z} U / N=k_{z}^{2} A / N$ is the Froude number of the primary wave, it can be alternatively defined from the amplitude of the horizontal velocity $U$ or the amplitude of the stream function and the vertical wavenumber of the primary wave, $F r$ equals here the amplitude $A$ since $k_{z}=1$ and $N=1, v$ is the viscosity introduced in the model and $R e$ is the Reynolds number based upon the horizontal velocity scale and the size of the domain, $R e=U L / v$

\begin{tabular}{lllrl}
\hline & Resolution & $F r=k_{z} U / N$ & \multicolumn{1}{l}{ Re } \\
\hline Run1 & $129^{3}$ & 0.256 & $3 \times 10^{-5}$ & $8.5 \times 10^{3}$ \\
Run2 & $513^{2}$ & 0.256 & $2.4 \times 10^{-5}$ & $1.1 \times 10^{4}$ \\
Run3 & $129^{2}$ & 0.256 & $5 \times 10^{-5}$ & $5.1 \times 10^{3}$ \\
Run4 & $513^{2}$ & 0.384 & $4 \times 10^{-5}$ & $9.6 \times 10^{3}$ \\
Run5 & $513^{2}$ & 0.448 & $4 \times 10^{-5}$ & $1.1 \times 10^{3}$ \\
Run6 & $129^{3}$ & 0.512 & $4 \times 10^{-5}$ & $1.3 \times 10^{3}$ \\
Run7 & $513^{2}$ & 0.512 & $4 \times 10^{-5}$ & $1.3 \times 10^{3}$ \\
\hline
\end{tabular}

linear stratification, of buoyancy frequency $N$. The numerical domain has an area equal to $\lambda^{2} / 4$ in the case of standing waves. Comparisons with oceanic or atmospheric flows can be made through a similarity in Froude number $\mathrm{Fr}$, and Reynolds number $R e . F r$ and $R e$ are defined from the primary wave parameters and background stratification: $k_{z} U / N$ and $R e=(U L / v)$, where $U$ is the amplitude of the horizontal velocity of the primary wave. We shall represent our results in terms of dimensionless quantities to facilitate comparisons. As an example of application of our simulations to an oceanic situation, let us consider an internal wave propagating in the thermocline, with $\mathrm{Fr}=$ 0.256 and $R e=1.1 \times 10^{4}$ (as for run 2 in Table 1 ). These parameters may characterize a wave with wavelength $\lambda=20 \mathrm{~m}$ and frequency $N / \sqrt{2}$ evolving in a background stratification $N=$ $2.5 \times 10^{-3} \mathrm{rad} / \mathrm{s}$. Note that the Reynolds number of this oceanic wave is slightly higher than that of the numerical simulation $\left(R e=3 \times 10^{4}\right.$ compared to $R e=1.1 \times 10^{4}$ ).

However, this difference in Reynolds numbers has no effect on the dynamics which is controlled by nonlinear wave-wave interactions and is therefore prescribed by the value of the Froude number. 


\section{Wave evolution toward breaking and induced turbulence}

The initial condition is chosen to be a standing wave, solution of the linear Boussinesq equations

$$
\begin{aligned}
\Psi(x, z) & =A \sin \left(k_{x} x\right) \sin \left(k_{z} z\right) \\
& =\frac{F r N}{k_{z}^{2}} \sin \left(k_{x} x\right) \sin \left(k_{z} z\right), \quad \rho^{*}=0 .
\end{aligned}
$$

It oscillates at the frequency $\omega$ given by the linear dispersion relationship $\omega=N \cos (\theta)$, where $\theta$ is the angle between the wave-vector and the horizontal plane. We choose $k_{x}=k_{z}=1$, so that $\omega=N / \sqrt{2}$. The nonlinearity of the primary wave is characterized by the Froude number $\mathrm{Fr}=\frac{k_{z} U}{N}$, where $U=k_{z} A$ is the amplitude of the horizontal velocity. Alternatively we could have defined a Froude number that corresponds to the maximum steepness of the isopycnals: $\frac{2 k_{k} k A}{\pi N}$. In the three- dimensional simulations, an additional wave perturbation with an amplitude equal to $\frac{1}{20} A$ and of wave vector $\vec{k}=(0,1,1)$ is introduced in order to favour the onset of fully three-dimensional wavebreaking.

Since a standing wave is only a solution of the linearized equations, the actual solution progressively departs from the linear approximation, involving weakly nonlinear interactions, followed by a breaking process (see Bouruet-Aubertot et al., 1995). The turbulent regime of interest is reached after wave breaking, (as discussed by BouruetAubertot et al., 1996), when energy is decaying by energy transfer towards small scales (Fig. 1). During this turbulent regime, local density inversions (overturning regions) coexist with strongly stratified regions.

Because of the anisotropy due to the stratification, we distinguish between spectra along the vertical and horizontal directions. Both display an

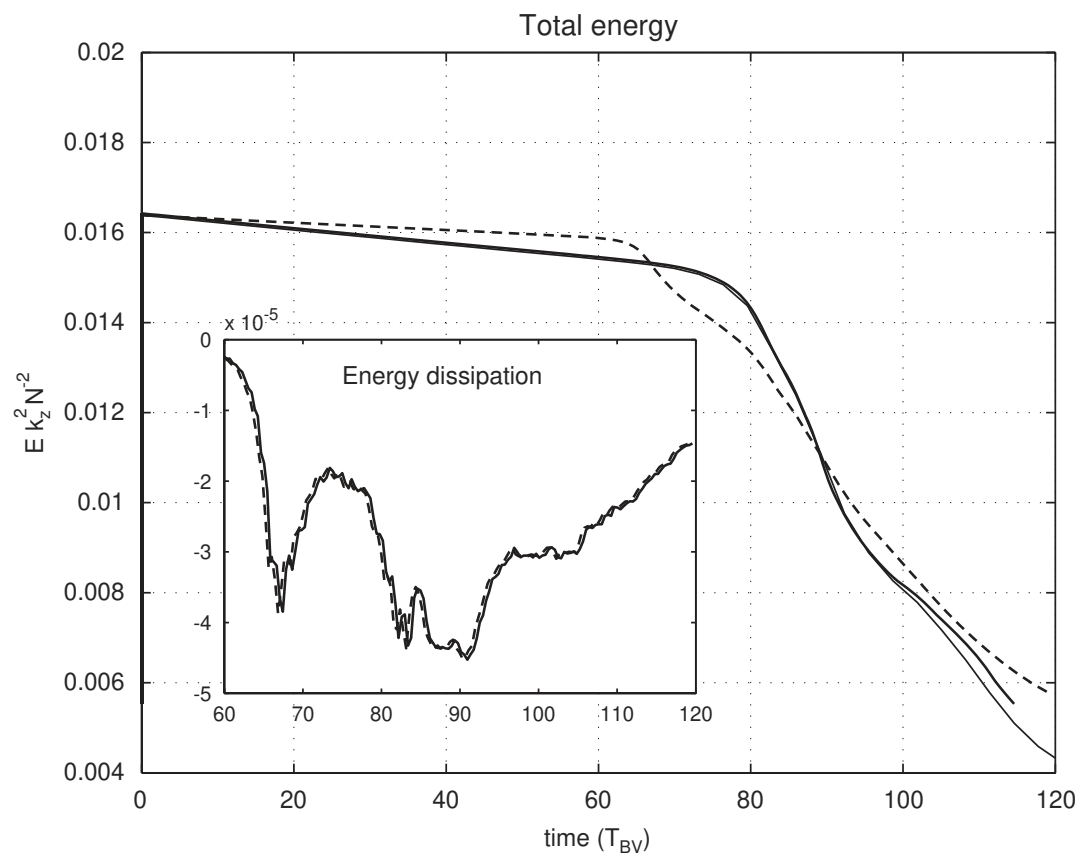

Fig. 1. Time evolution of the total energy (kinetic energy + available potential energy), $E=0.5\left[|\vec{v}|^{2}+g^{2} /\left(\rho_{0} N\right)^{2} \rho^{\prime 2}\right]$, for an initial wave with a Froude number $F r=0.256$ ( $E$ is represented in a dimensionless form: $E k_{z}^{2} N^{-2}$ ). The three-dimensional calculation is represented with a thick bold line (run1) while the two-dimensional calculations are represented with a thick dashed line for the $512^{2}$ resolution (run2) and with a thin line for the $128^{2}$ resolution (run3). The analysis is focused on the time interval [83T $\left.T_{B V}, 92 T_{B V}\right]$ corresponding to a total energy within $[60 \% E(t=0), 80 \% E(t=0)]$. Time evolution of the energy time derivative is represented in a sub-figure (black line) as well as the dissipation rate computed directly (dashed line). 
inertial range with a spectral slope close to -3 . It was shown by Bouruet-Aubertot et al. (1996) that the spectral energy at a wavenumber $k_{z}$ (calculated along the vertical direction) saturates at a level $\alpha N^{2} k_{z}^{-3}$ and does not depend on the initial condition. A relevant scale for this buoyancy subrange is the Ozmidov's scale $\lambda_{0}$, defined from the rate of energy dissipation $\varepsilon$ by

$\lambda_{0}=\left(\frac{\varepsilon}{N^{3}}\right)^{1 / 2}$.

We calculate $\varepsilon$ as the total energy drop $\Delta E$ (per unit of volume) during the time of analysis $\Delta t$ (namely $E \approx[60 \% E(t=0), 80 \% E(t=0)]), \quad \varepsilon=$ $-(\Delta E / \Delta t)$.

An example of the energy decrease is shown in Fig. 1 for the smallest wave Froude number $(F r=$ 0.256). Three different simulations are shown: a low resolution three-dimensional simulation, the corresponding two-dimensional simulation with the same resolution and a high resolution twodimensional simulation. The first two curves are very close which demonstrates that overall nonlinear energy transfers have the same global effect on the total energy though details in kinetic and potential energy transfers might differ between the two-dimensional and the three-dimensional case. The two-dimensional higher resolution case matches the two other cases closely as well, the main difference arising from the viscosity introduced in the model (see Table 1): the onset of wavebreaking occurs earlier in the latter case (2D high resolution) because of the smaller viscosity of the simulation. Later on, during the time interval of interest, the total energy decreases in a very similar way in all three cases. It results from non linear energy transfers that lead to wavebreaking. Because these transfers are set at large scales by the instability of the primary wave, the resulting energy decrease is not strongly modified whether two-dimensional or three-dimensional simulations are considered. In contrast fully $3 \mathrm{D}$ dynamics is obtained when the large scale primary wave is statically unstable as shown by Koudella (1999). This finding is well predicted by Floquet stability analysis (Klostermeyer, 1991; Lombard and Riley, 1996) which shows that $2 \mathrm{D}$ perturbations are more unstable than 3D perturbations so far as the primary wave is statically stable.

The rate of energy decay $\varepsilon$ is plotted in Fig. 2 versus the initial wave Froude number $\mathrm{Fr}$. The Ozmidov scale $\lambda_{0}=\left(\varepsilon / N^{3}\right)^{1 / 2}$ is represented. Its variation is consistent with a law in $\mathrm{Fr}^{3 / 2}$ ( $\varepsilon$ in $\mathrm{Fr}^{3}$ ). We notice again that the two-dimensional computations (empty circles) are in good agreement with the three-dimensional ones (filled circles).

Therefore, we analyze both types of simulations, the three-dimensional case allows us to investigate the realistic structure of events observed during wavebreaking, while the high resolution twodimensional simulations enable us to investigate intermittency at small vertical scales and provide a link with mixing processes.

\section{Evidence for strongly stratified sheets}

\subsection{Histograms of density differences at different vertical scales}

We compute the histograms (probability distribution functions, pdf) of density differences, $(\rho(z+\Delta z)-\rho(z)) / \Delta z$ for different vertical scales, $\Delta z$. Thus, neutral stratification corresponds to the zero abscissa in the figures and the mean stratification corresponds to -1 . These pdfs are displayed in Fig. 3 for different $\Delta z$ and for two Froude numbers of the wave, $F r=0.256$ and 0.512 . Both three- and two-dimensional simulations are shown. Note that a comparison between these cases is made for similar vertical separations in terms of units of the Ozmidov length scale. This is consistent with the fact that the shape of the pdfs evolves as a function of the vertical separation as a different dynamical regime is concerned (from weakly nonlinear waves at large scales to breaking waves at smaller scales).

We notice the obvious asymmetry (strong skewness) of the pdf at small scales (Figs. 3A and $\mathrm{C}$ ), which reflects the difference between stably stratified regions (left side of the curves) and overturned regions (right side). On the left side, long tails are obtained at small scales, corresponding to highly stratified sheets: a plateau is almost reached (Figs. 3A and B), with local 


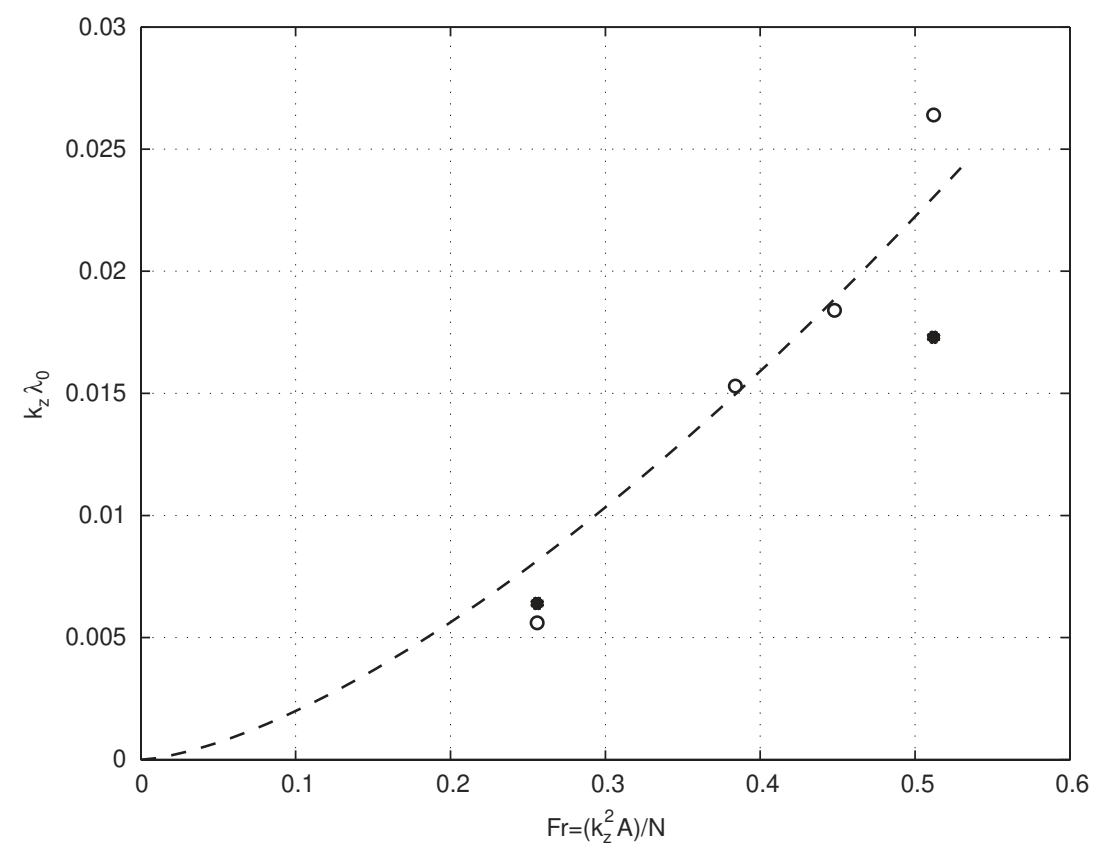

Fig. 2. Ozmidov length scale $\lambda_{0}=\left(\varepsilon / N^{3}\right)^{0.5}$ as a function of wave Froude number $F r$, 2D simulations are represented with empty circles and 3D simulations with filled circles. A $\mathrm{Fr}^{3 / 2}$ fit is represented with a dotted line.

stratification reaching up to thirty times the initial background stratification (Fig. 3C). This asymmetry decreases as the vertical scale increases (Figs. 3B and D). Very similar results are obtained for the three-dimensional simulations especially when the primary wave is of small amplitude (Figs. 3A and $\mathrm{B})$. This results from the fact that the dynamics remains almost two-dimensional even during wavebreaking (e.g. Fig. 3B and BouruetAubertot et al., 2001). When the wave is of higher amplitude slight differences can be noticed (Figs. $3 \mathrm{C}$ and D) especially for strongly stratified regions. These differences can be attributed to convective instabilities that occur in the transverse plane in the three-dimensional simulations, as will be discussed in the next section.

In order to measure the probability of occurrence of overturning events, we have computed the relative volume of unstable (overturned) regions compared to the total volume of fluid. This quantity is displayed as a function of the vertical scale $\Delta z$ in Fig. 4. The plot underlines the fact that breaking events are localized in space (small vertical extent) and rare (a maximum of $18 \%$ is reached for the highest wave amplitude). We expect that the strong stable density gradients are produced at the edge of regions mixed by local overturning. We check this assumption in the next section by a direct examination of the most intense events.

\subsection{Dynamical processes associated with strongly stratified regions}

We have selected the most intense events characterized by extreme stable density gradients (at scale $\Delta z \leqslant 10 \lambda_{0}$ ). They appear as thin horizontal sheets surrounded by mixed convective zones. An example is visualized in Fig. 5 by means of maps of density and vertical density gradient. In the plane $(x, z)$ of the primary wave the most strongly stratified region is located around $x \approx[2.5,3.14]$ and $z \approx 0.5$ (Figs. 5A and $\mathrm{C}$ ), with a vertical density gradient up to 15 times the background value. We notice local density overturning on both sides of this stratified sheet (visualized by dark 

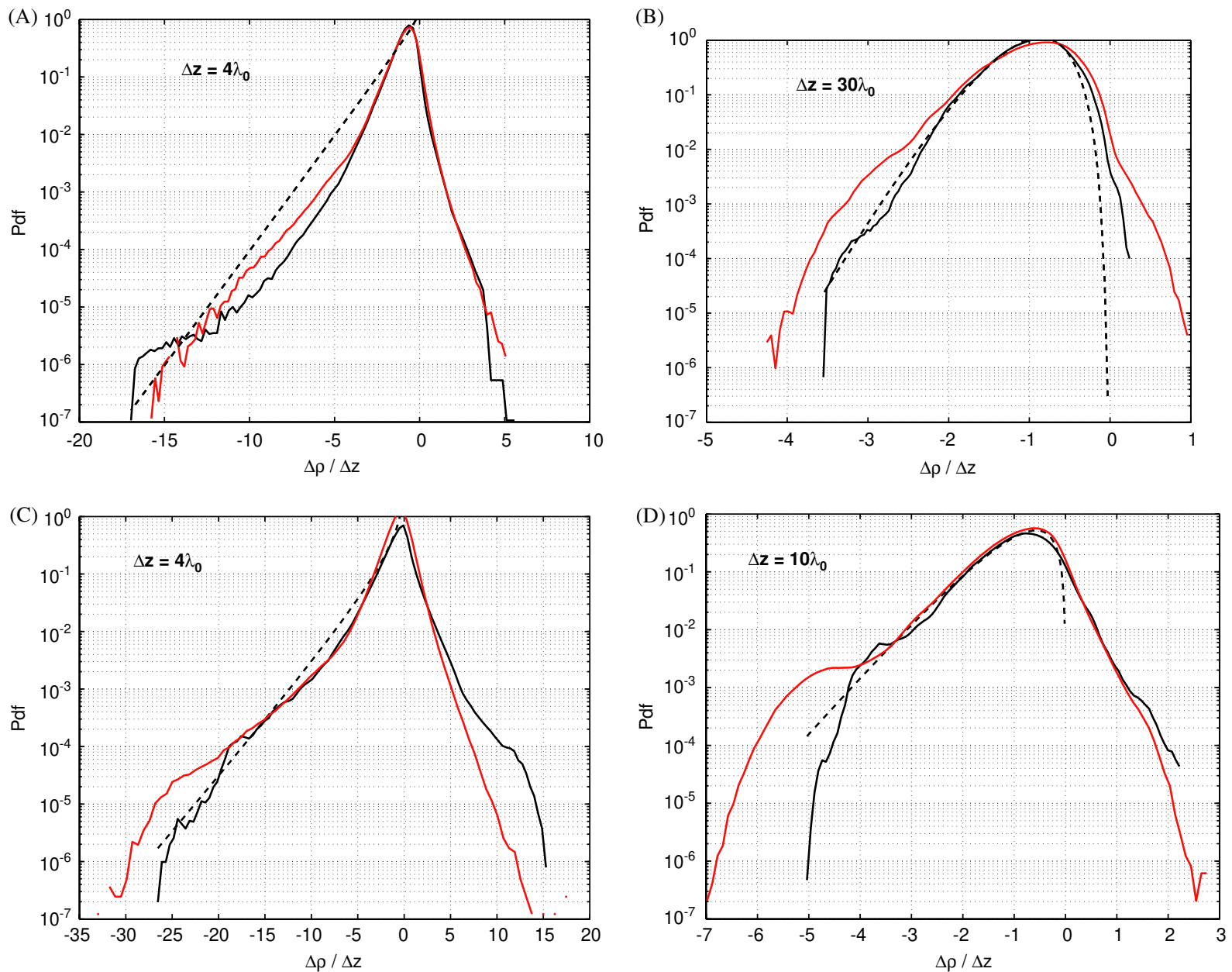

Fig. 3. Probability density function of the density gradient at different vertical scales $\operatorname{pdf}([\rho(x, z+\Delta z)-\rho(x, z)]) / \Delta z$ : (A) $\Delta z=4 \lambda_{0}$ (i.e. $\Delta z=4 \mathrm{~d} z$ ), $F r=0.256$ (runs 1 and 2), (B) $\Delta z=30 \lambda_{0}$ (i.e. $\Delta z=32 \mathrm{~d} z$ ), $F r=0.256$ (runs 1 and 2), (C) $\Delta z=\lambda_{0}$ (i.e. $\Delta z=4 \mathrm{~d} z$ for the 3D simulation, and $\Delta z=8 \mathrm{~d} z$ for the 2D simulation), $F r=0.512$ (runs 6 and 7), (D) $\Delta z=10 \lambda_{0}$, (i.e. $\Delta z=32 \mathrm{~d} z$ for the 3D simulation, and $\Delta z=48 \mathrm{~d} z$ for the 2D simulation), $F r=0.512$ (runs 6 and 7), where $\mathrm{d} z=\pi / 512 k_{z}^{-1}$ is the grid cell of the two-dimensional simulation. Three-dimensional results are represented in gray and two-dimensional results in black. Theoretical prediction of the Hayes et al.'s model is represented with a dashed line (with $\mu=37 k_{z}=0.2 \lambda_{0}^{-1}$ in (A) and (B) and $\mu=8.3 \mathrm{kz}=0.2 \lambda_{0}^{-1}$ in (C) and (D)).

gray patches in Fig. 5C). In fact, the existence of the sheet is correlated with breaking events: intense vortices associated with wavebreaking "dig" a local step in the density profile. For standing waves, these most intense events occur near the edge of the domain. However, similar association of a mixing vortex and a stratified sheet is also obtained for propagating waves in a periodic domain (e.g. Fig. 12 from Bouruet-Aubertot et al., 2004). The sheet has a wide extent in the $y$ direction normal to the primary wave (Figs. 5B and $\mathrm{D}$ ). We, therefore, conclude that the shape of these sheets is largely controlled by the dominant primary wave, even in the presence of significant $3 \mathrm{D}$ perturbations.

Strongly stratified sheets are still enhanced when the amplitude of the wave is increased (see Fig. 6), the extremum vertical density gradient is significantly stronger than in the previous case $(-26$ in Fig. $6 \mathrm{D}$ to be compared with -15 in Fig. 5D). 


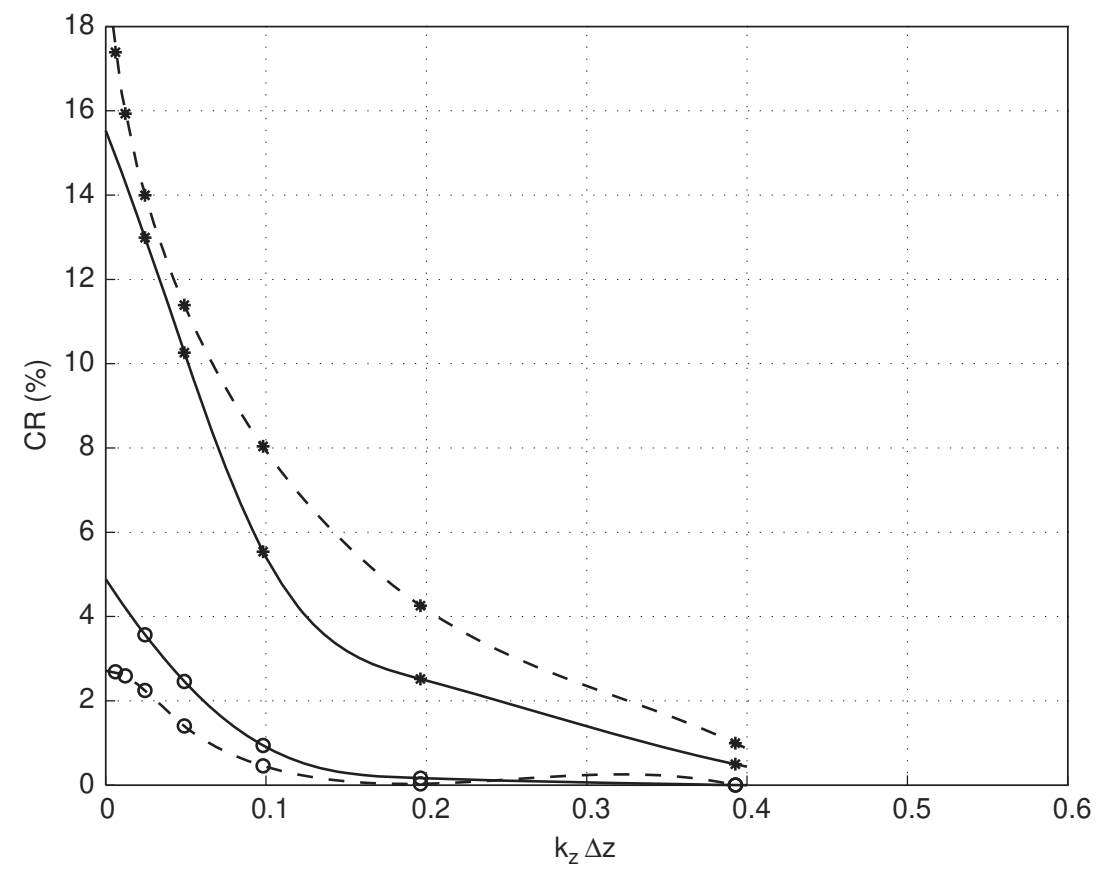

Fig. 4. Proportion of convective regions as a function of the vertical scale, for two values of the Froude number: $F r=0.512$ (filled circles, runs 6 and 7) and $F r=0.256$ (empty circles, runs 1 and 2). The 2D calculations are represented with a dashed line and the 3D calculations with a continuous line. Only four curves are represented for clarity.

Convective instabilities are then clearly seen in the transverse direction (Fig. 6B), therefore overturning events are more efficient in building up the step, as previously noticed in Figs. 3C and D.

The vertical extent of the sheet is small, about a few Ozmidov length scales: $7.26 \times 10^{-2}$ for the two cases in Figs. 5 and 6, i.e. $5 \lambda_{0}$ for $F r=0.512$ and $12 \lambda_{0}$ for $F r=0.256$. In order to investigate the persistence of these structures we have introduced a forcing such that the energy of the primary wave is kept constant. Sheets result from independent wavebreaking events and their persistence is limited in time, lasting for a few buoyancy periods (see Bouruet-Aubertot et al., 2004).

In summary, the density gradients are strongly intermittent at small vertical scales, with a strong asymmetry between stable and convective regions. The intermittency results from breaking events that modify the vertical density structure into a succession of turbulent convective regions and strongly stratified sheets. The main question we address in the next section is whether any information on mixing can be inferred from these pdfs.

\section{Link with vertical mixing}

\subsection{A model for the density gradient histograms}

Two processes may be responsible for the increased skewness of the pdf of density gradients at small vertical scale, either the reversible straining produced by internal gravity waves or irreversible mixing events. These two alternatives were addressed in theoretical models. The effect of reversible straining has been considered by Desaubies and Gregg (1981). Assuming Gaussian statistics for the vertical wave displacement, they find a non Gaussian statistics for the difference of displacements. It is more and more skewed as the distance is reduced, as we expect for intermittency. The second model is the gamma pdf, proposed by Hayes et al. (1975), who assume a staircase density 

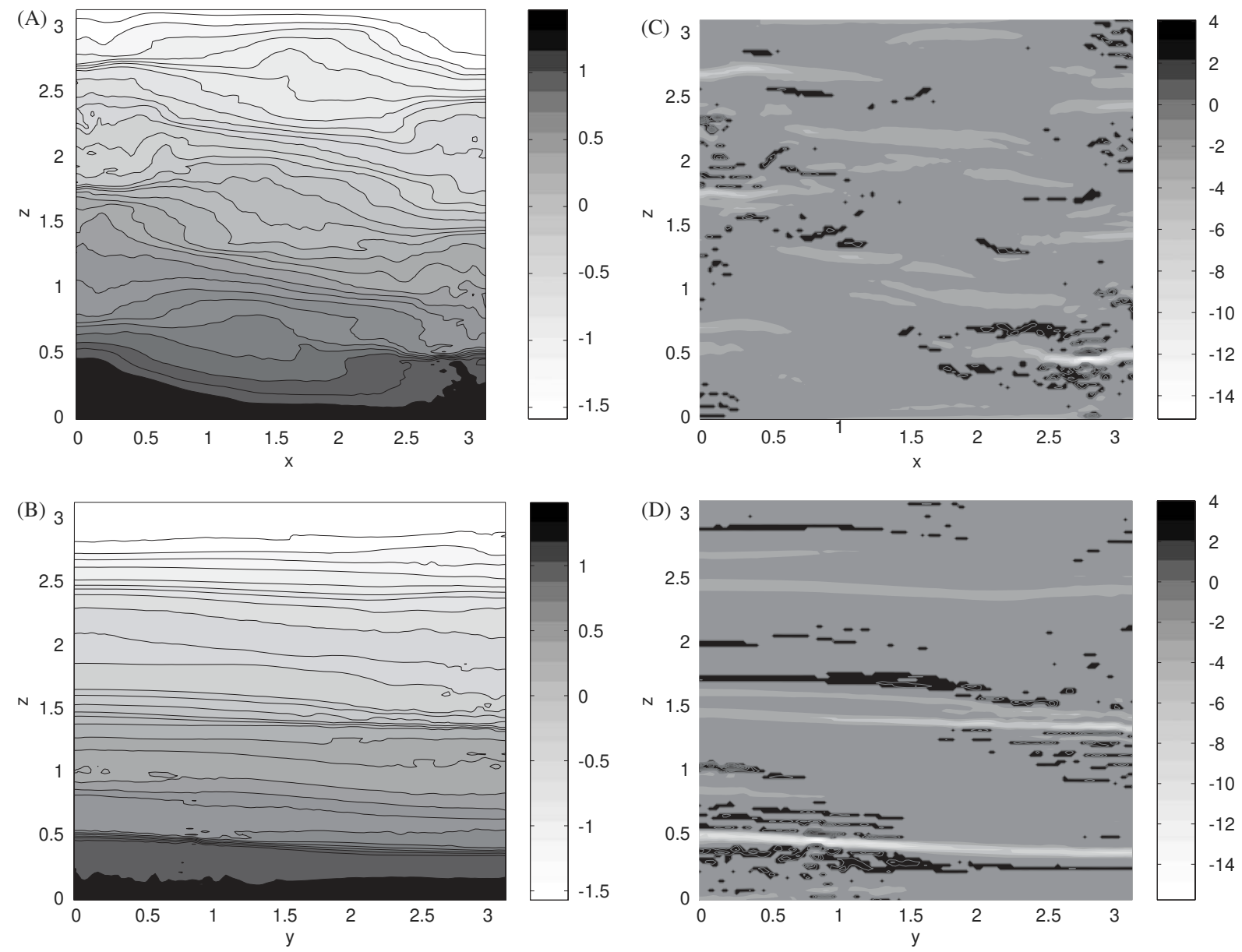

Fig. 5. Strongly stratified sheets with an initial dominant primary wave $\vec{k}=(1,0,1), F r=0.256$ (run 1 in Table 1). The density fields (top) and vertical density gradients (bottom) are represented in two perpendicular vertical planes crossing at the location of extremal vertical density gradient. (A) Density field in the $(x, z)$ plane at $y=0.83$. (B) Density field in the $(y, z)$ plane at $x=2.79$. (C) Vertical density gradient in the $(x, z)$ plane at $y=0.83$. (D) Vertical density gradient in the $(y, z)$ plane at $x=2.79$. We notice that the strongly stratified sheets (white) are surrounded by convective zones (unstable density gradients represented in dark gray).

profile with randomly distributed steps separating mixed layers with uniform density. This model provides a good fit to oceanic data in the range of vertical scales $(1-10 \mathrm{~m})$, but it clearly implies excessive mixing. However the gamma pdf, obtained as a sum of statistical variables with Poisson statistics, can be justified in more general terms. It is infinitely divisible, meaning that a sum of independent random variables with this statistics has the same statistics (like for a Gaussian). Later Pinkel and Anderson (1992) considered a more realistic density profile since they allow partial mixing between sheets, assuming layers with random gradients. Interestingly, because of the above property of the gamma pdf, the pdf model for density differences has the same analytical expression (Eq. (16)) than that predicted by Hayes et al. (1975).

This model provides also a reasonable fit to our numerical data at scales smaller than $30 \lambda_{0}$ (see Bouruet-Aubertot et al., 2004). It does not match with the weakly nonlinear regime at larger scales, 

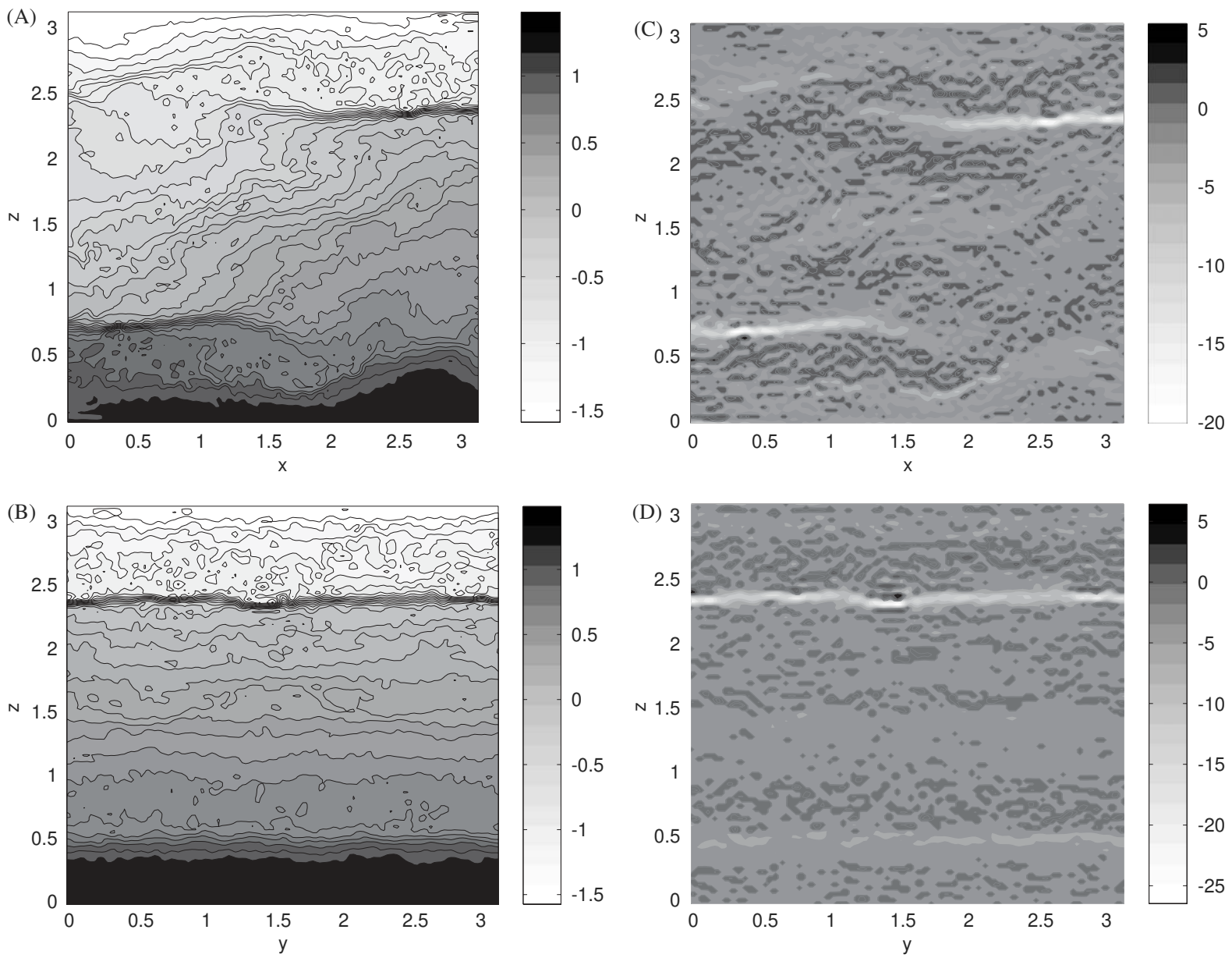

Fig. 6. Strongly stratified sheets in a three-dimensional simulation, with a primary wave of high amplitude $F r=0.512$ (run6 in Table 1). The density fields (top) and vertical density gradients (bottom) are represented in two perpendicular vertical planes crossing at the location of extremal vertical density gradient. (A) Density field in the $(x, z)$ plane at $y=1.32$. (B) Density field in the $(y, z)$ plane at $x=2.57$. (C) Vertical density gradient in the $(x, z)$ plane at $y=1.32$. (D) Vertical density gradient in the $(y, z)$ plane at $x=2.57$. We notice that the strongly stratified sheets (white) are surrounded by convective zones (unstable density gradients represented in dark gray).

for which the model of Desaubies and Gregg (1981) is found more appropriate. However mixing effects are controlled by the smaller scales, for which the gamma pdf model is appropriate. The probability density of the density difference $\Delta \rho$ at separation $\Delta z$ is given by (see Eq. (9) of Hayes et al.)

$$
P_{\mathrm{g}}(\Delta \rho \mid \Delta z)=\frac{\mu}{\beta \Gamma(\mu \Delta z)}\left(\mu \frac{\Delta \rho}{\beta}\right)^{\mu \Delta z-1} \exp \left(-\mu \frac{\Delta \rho}{\beta}\right)
$$

where $\Gamma$ represents the Gamma function and $\beta$ the mean density gradient (equal to -1 in our computations). The distribution at all scales $\Delta z$ is determined by the single parameter $\mu$. In the model of Hayes et al. (1975) $\mu$ denotes the mean number of steps per unit of height.

One easily checks that the integral of this probability density is unity and that the moment of degree $n$ is $\left\langle(\Delta \rho)^{n}\right\rangle=\int_{0}^{\infty} P(\Delta \rho)(\Delta \rho)^{n} d(\Delta \rho)=$ $(\beta / \mu)^{n}(\mu \Delta z)(\mu \Delta z+1) \cdots(\mu \Delta z+n-1)$. In particular the mean $n=1$ is equal to the mean gradient $\beta$, 
as it should. The variance of the fluctuations is $\left\langle(\Delta \rho-\langle\Delta \rho\rangle)^{2}\right\rangle=(\beta / \mu)^{2} \mu \Delta z$. The density fluctuations can be considered as resulting from a "random walk" with steps $\beta / \mu$. The variance is proportional to the mean number of steps $\mu \Delta z$. Note that the third-order moment for density fluctuations varies as well linearly with the vertical separation: $\left\langle(\Delta \rho-\langle\Delta \rho\rangle)^{3}\right\rangle=2(\beta / \mu)^{3} \mu \Delta z$. These relations provide a first test of the model, and a fit of its parameter $\mu$. We have plotted these two moments as a function of the vertical distance $\Delta z$ in Fig. 7. It can be reasonably fitted by a linear relationship in a limited range. The mean distance between sheets $\mu^{-1}$, is inferred from the corresponding slopes ( $\beta=-1$ in our computations).

Once the single parameter $\mu$ has been determined, the probability distribution at different scales is determined by the model, which provides a severe test (see Fig. 16 Bouruet-Aubertot et al., 2004 for comparison). The comparison shown in Fig. 3 is fairly good for vertical separations $\Delta z \approx$ $\left[\lambda_{0}, 30 \lambda_{0}\right]$. Nevertheless, the tails of the gamma pdf model tend to an exponential at small separations, while flatter tails are observed: intermittency is underestimated by the model (Figs. 3A and D). Furthermore, the occurrence of convective zones with positive $\Delta \rho$ is ignored.

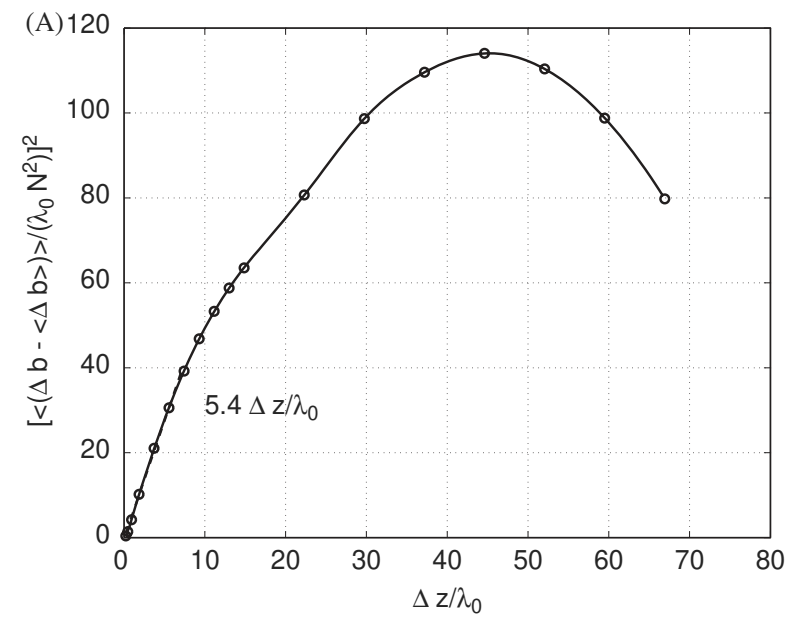

\subsection{An indirect estimate of energy dissipation and mixing}

The formation of sheets and layers is clearly associated with energy dissipation and vertical mixing. We, therefore, expect a link between the Ozmidov scale, related to energy dissipation, and the mean vertical distance between sheets, $\mu^{-1}$. Fig. 8 shows indeed that these two quantities are proportional $\mu^{-1} \approx 5.3 \lambda_{0}$.

We next computed the vertical eddy diffusivity $K_{\mathrm{d}}$ using the method proposed by Winters and D'Asaro (1996) that leads to the following expression for the Cox number:

$$
\operatorname{Cox}=\frac{K_{\mathrm{d}}}{\kappa}=\frac{\left\langle\|\nabla \rho\|^{2}\right\rangle_{I}}{\left(\mathrm{~d} \rho_{b} / \mathrm{d} z\right)^{2}},
$$

where \langle\rangle$_{I}$ denotes an average over an isopycnal surface and $\rho_{b}$ refers to the sorted density profile. The key difference with the Osborn and Cox (1972) expression for the Cox number is that the average of the squared density gradient is computed over an isopycnal surface in place of a horizontal plane and the vertical gradient of the sorted density profile is taken into account in place of the horizontal mean of the vertical density

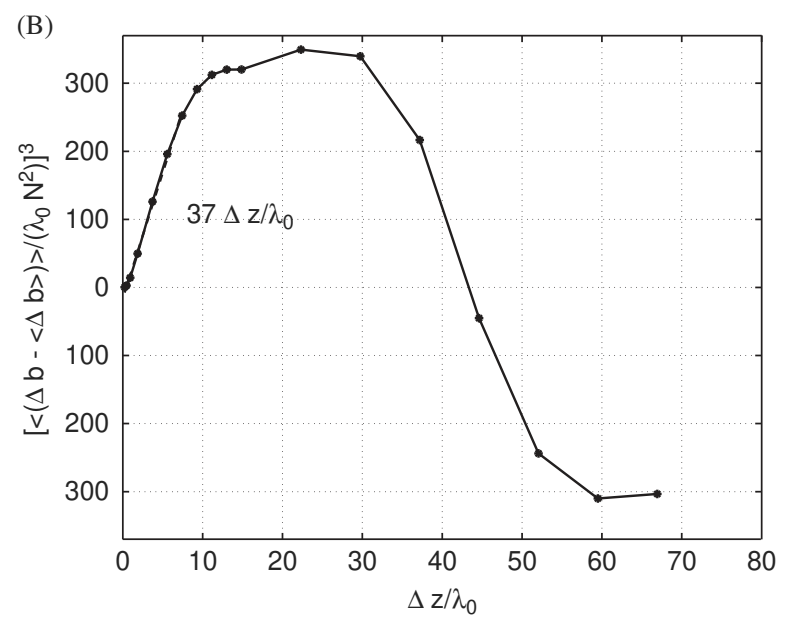

Fig. 7. (A) Variance of reduced density differences $\Delta b=g / \rho_{0}(\rho(x, z+\Delta z)-\rho(x, z))$ (in units of $\left(\lambda_{0} N^{2}\right)$ as a function of vertical separation $\Delta z$ (in units of $\lambda_{0}$ ) (run7), the slope at small scales is equal to $\beta^{2} / \mu$ (see text), i.e. $1 /\left(\mu \lambda_{0}\right)$ in dimensionless form. (B) $\left\langle[\Delta b-\langle\Delta b\rangle]^{3}\right\rangle$ (in units of $\left(\lambda_{0} N^{2}\right)^{3}$ ) as a function of $\Delta z$ (in units of $\lambda_{0}$ ) (run7), the slope at small scale is equal to $2 \beta^{3} / \mu^{2}$ (see text), i.e. $2 /\left(\mu \lambda_{0}\right)^{2}$. The sheet spacing $\mu^{-1}$ is inferred from a linear fit of the two curves for vertical separation within $\left[0.2 \lambda_{0}, 7.5 \lambda_{0}\right]$, a value of $5.4 \lambda_{0}$ is obtained. 


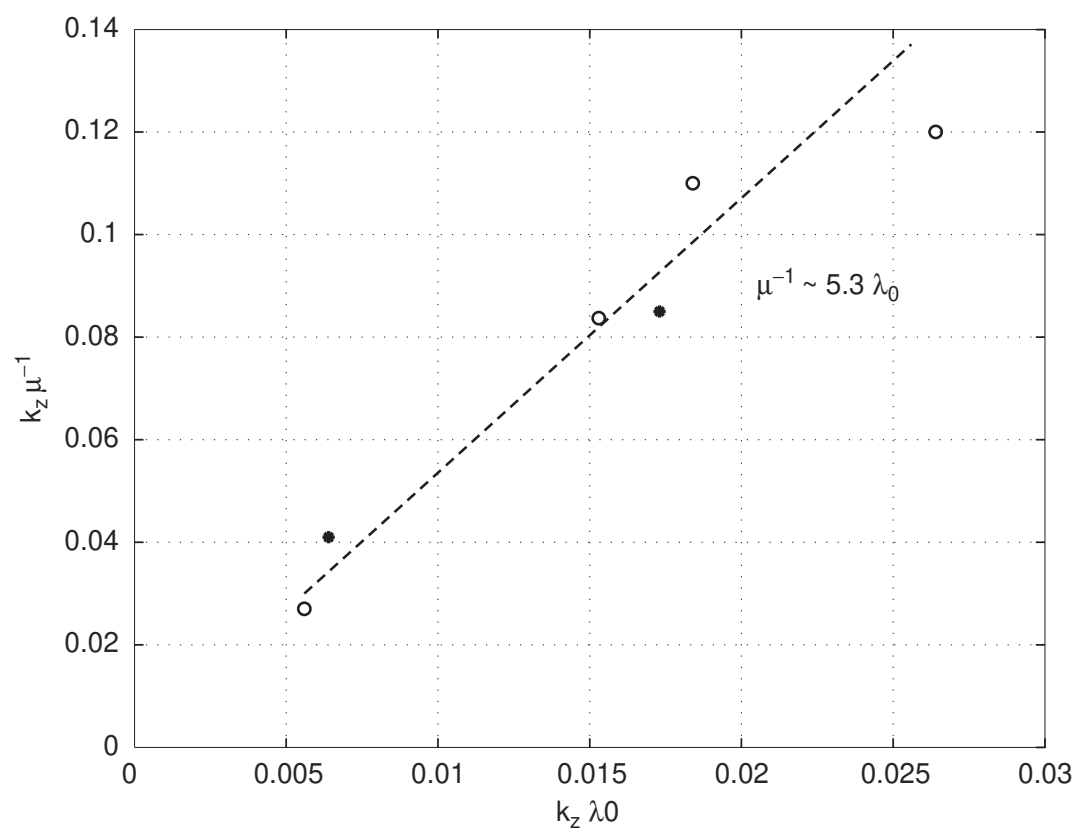

Fig. 8. Mean distance between steps $\left(\mu^{-1}\right)$ as a function of the Ozmidov length scale $\left(\lambda_{0}=\left(\varepsilon / N^{3}\right)^{1 / 2}\right)$, 2D simulations are represented with empty circles and $3 \mathrm{D}$ simulations with filled circles.

gradient. The drawback of the latter method is that it overestimates the Cox number when the mean vertical density gradient becomes small. We found indeed that while an excellent agreement between the two methods was obtained for the smallest wave amplitude, a discrepancy of a factor of 2 appears for the highest wave amplitude. Note also that in the method of Thorpe, a sorting of the fluid parcels is used along a vertical line of measurement, so it can be viewed as a simplified version of the method of Winters and D'Asaro suitable for field data.

The vertical eddy diffusivity $K_{\mathrm{d}}$ associated with wave breaking is classically related to energy dissipation, following Osborn (1980). Indeed the vertical mixing requires an increase of potential energy, to lift the heavier fluid. Part of the wave energy decrease corresponds to a conversion to this background potential energy. This results in a relationship

$K_{\mathrm{d}}=\frac{\gamma}{\gamma+1} \frac{\varepsilon}{N^{2}}$ where $\gamma /(\gamma+1) \leqslant 1$ is a mixing efficiency. We plot $K_{\mathrm{d}} / \kappa$ versus $\varepsilon / v N^{2}$ in Fig. 9. The two quantities are indeed proportional, with a similar result for two- and three-dimensional computations. The slope determines a high mixing efficiency $\gamma /(\gamma+$ $1)=0.7$ which deserves further comments. Indeed estimates in the ocean and in some laboratory experiments lead to a smaller mixing efficiency, about 0.2. Nevertheless, our result is more consistent with that inferred from laboratory experiments by Barry et al. (2001) who found that

$\frac{K_{\mathrm{d}}}{\kappa^{1 / 3} v^{2 / 3}}=0.65 \frac{\varepsilon_{\mathrm{ke}}}{v N^{2}}$

for turbulence intensity $\left(\varepsilon_{\mathrm{ke}} / v N^{2}\right)$, within the interval $[10,300]$. This agrees with our simulations at Prandtl number $\operatorname{Pr}=(v / \kappa)=1$, but yields lower values for smaller $P r$. Indeed mixing efficiency in similar computations was found to decrease with increasing Prandtl number, reaching a value of 0.3 for $P r=10$ (Bouruet-Aubertot et al., 2001). In laboratory experiments, using salt concentration, $\operatorname{Pr}=1000$, so that a still smaller 


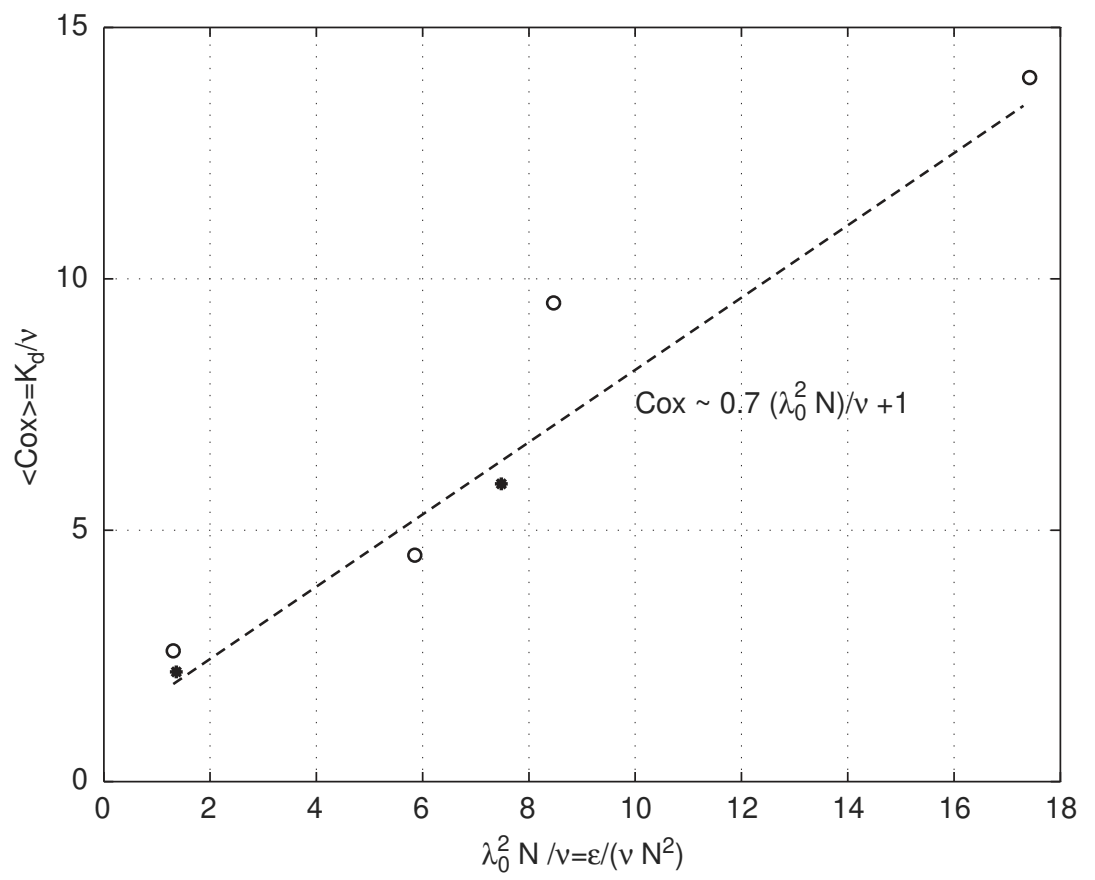

Fig. 9. Mean Cox number as a function of dimensionless total energy dissipation $\lambda_{0}^{2} N / v, 2 \mathrm{D}$ simulations are represented with empty circles and 3D simulations with filled circles.

mixing efficiency is expected. We expect that such Prandtl number dependency is most important at the lowest values of the turbulent intensity.

Combining this result with the link between $\lambda_{0}$ and $\mu^{-1}$, we can relate the vertical eddy diffusivity with $\mu$. In the next section we shall perform a similar analysis of the vertical density gradients to oceanic data and deduce estimates of energy dissipation $\varepsilon$ and vertical eddy diffusivity $K_{\mathrm{d}}$.

\section{Application to in situ oceanic measurements in an area of energetic internal tides}

We have analyzed a set of 24 temperature and salinity profiles collected during the ARCANE experiment in 1996 at a fixed point, $\left(42^{\circ} 06^{\prime} \mathrm{N}\right.$, $9^{\circ} 33^{\prime} \mathrm{W}$ ), located on the continental slope west of the Iberian peninsula (Le Cann et al., 1998). These profiles were acquired every hour during one day and the effective vertical resolution is equal to $1 \mathrm{~m}$. A temperature salinity diagram of these data is shown in Fig. 10. Surface waters are characterized by temperatures greater than $13.5^{\circ}$ and a mean salinity of $35.9 \mathrm{psu}$. Deeper, North-Atlantic central water $(\mathrm{NACW})$ is represented by $[\mathrm{AB}]$ while point $\mathrm{C}$ corresponds to Mediterranean water (MW). The line $\mathrm{BC}$ results from mixing between $\mathrm{MW}$ and some water mass formed by a subduction process further North (point B). Point D represents Labrador sea deep water (LSW) and the region between C and D is characterized by salt-fingering processes.

The corresponding mean profiles of potential density $\sigma$, temperature $T$ and salinity $S$, are represented in Fig. 11. Below the thermocline the density profile is almost linear down to about $-1200 \mathrm{~m}$ following which the stratification is weaker. In the interior region, for depth within $[-1000 \mathrm{~m},-400 \mathrm{~m}]$, the temperature is almost constant (Fig. 10B) and the stratification is due mostly to salinity in this region (Fig. 11C). We restrict our analysis in this depth interval.

In this region the dynamics is characterized by the presence of a strong baroclinic M2 tide 


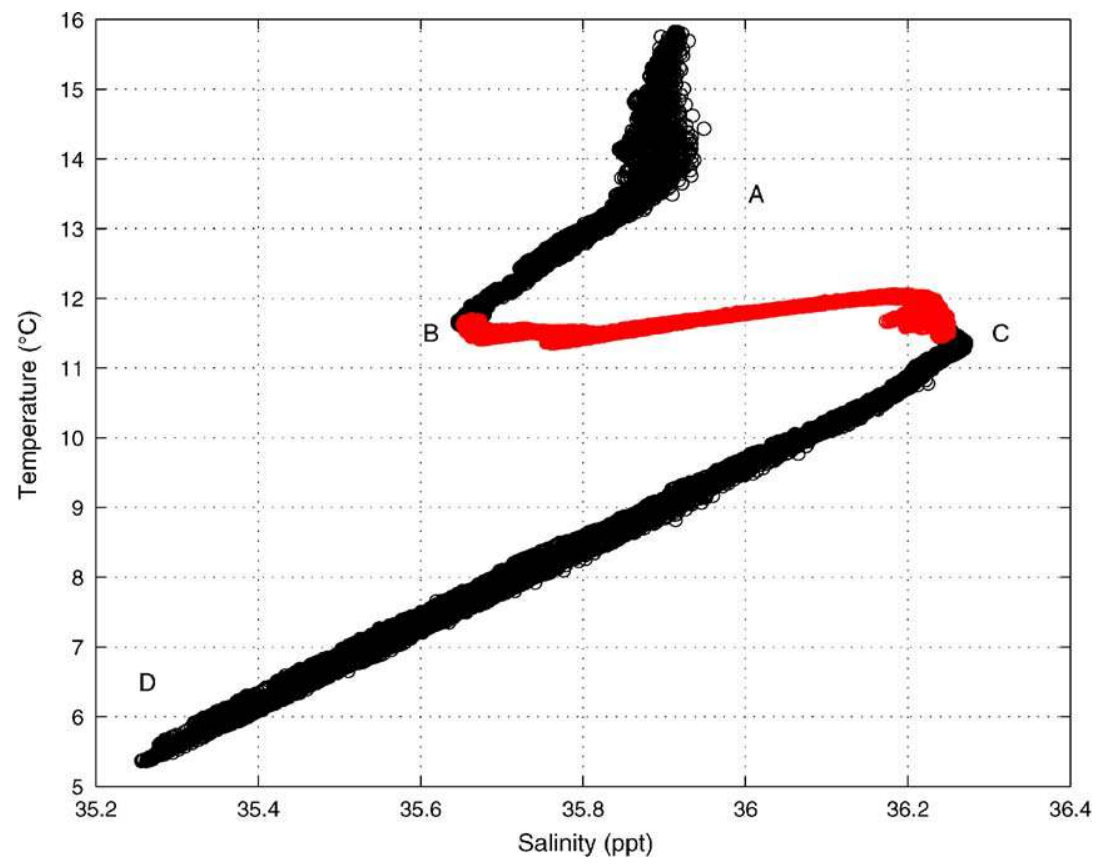

Fig. 10. Temperature salinity diagram taken from repeated CTD profiles at $\left(42^{\circ} 06^{\prime} \mathrm{N}, 9^{\circ} 33^{\prime} \mathrm{W}\right)$ west of the Iberian peninsula during the ARCANE experiment. The analysis is focused on the data points represented in grey (red in online version) and corresponding to depth within $[-1000 \mathrm{~m},-400 \mathrm{~m}]$.
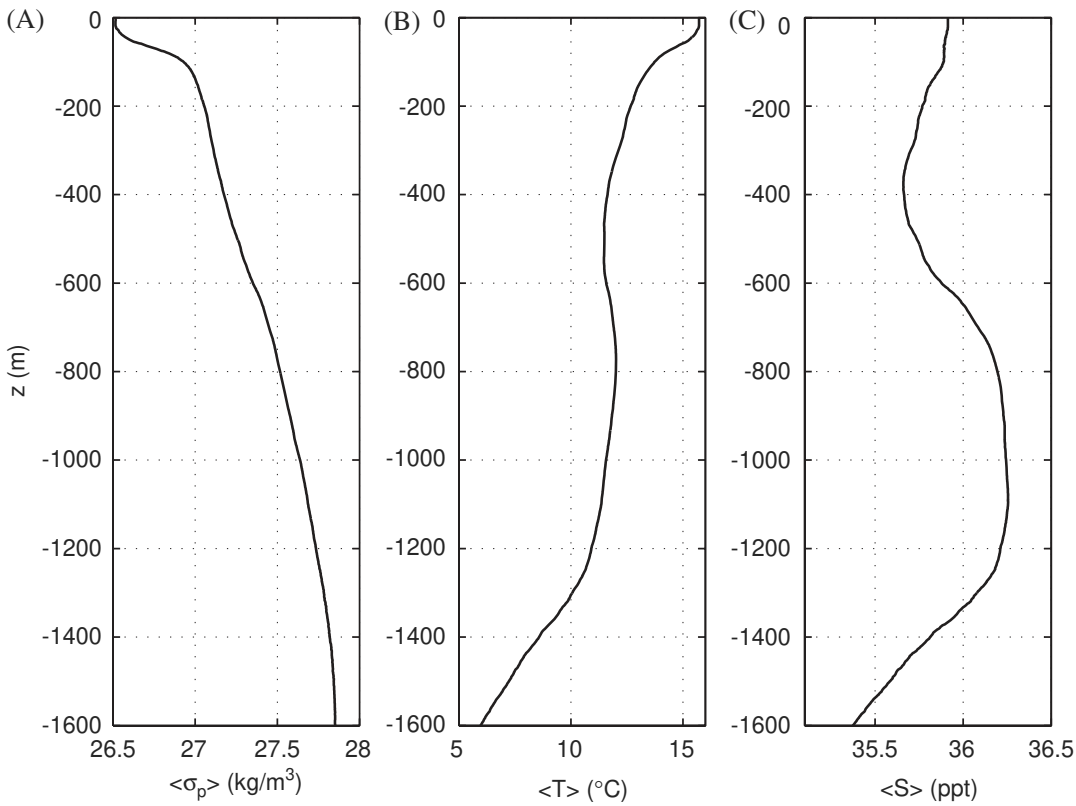

Fig. 11. Temporal mean of the vertical profiles: (A) potential density $\sigma_{p},(\mathrm{~B})$ temperature $T,(\mathrm{C})$ salinity $S$. Variances of $\sigma_{p}, T$ and $S$ are, respectively, equal to $2.3 \times 10^{-4}\left(\mathrm{~kg} / \mathrm{m}^{3}\right)^{2}, 3.0 \times 10^{-3}\left({ }^{\circ} \mathrm{C}\right)^{2}$ and $5.5 \times 10^{-4}$ for depth within $[-1000 \mathrm{~m},-400 \mathrm{~m}]$. 


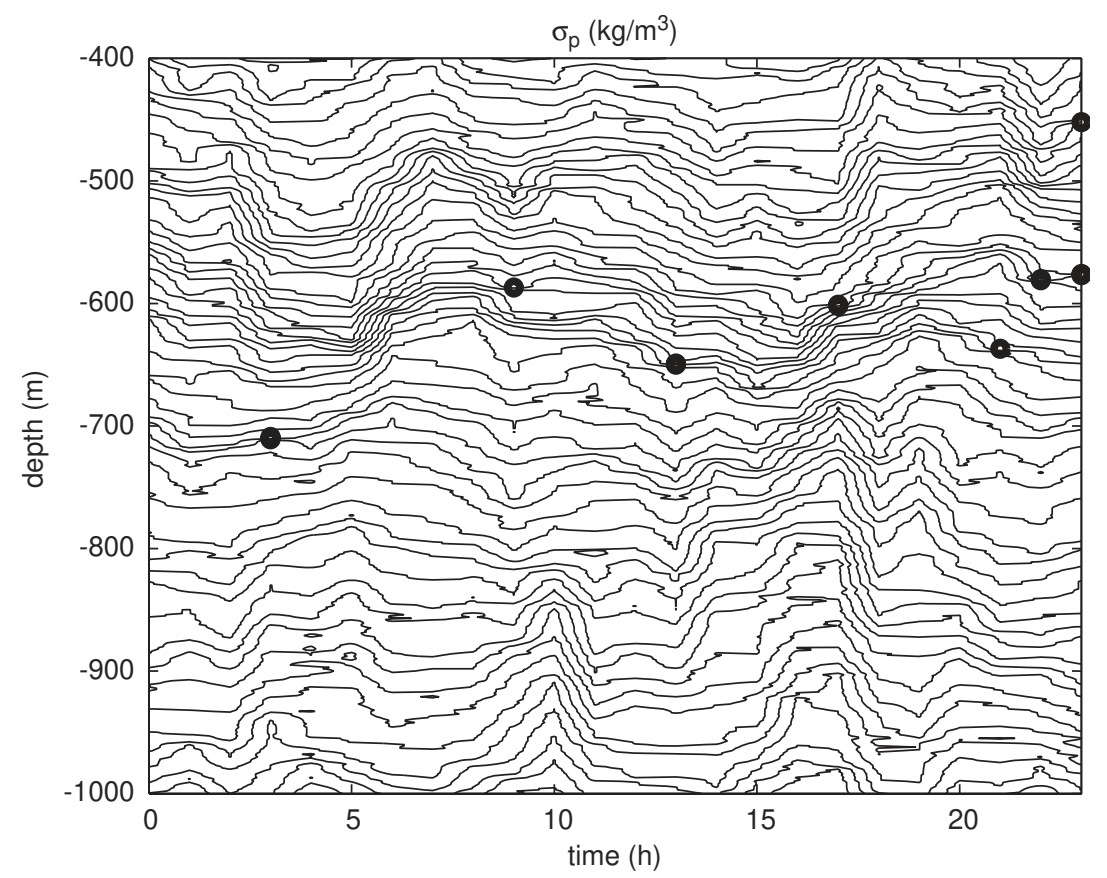

Fig. 12. Time depth map of potential density inferred from the 24 CTD profiles for depth within $[-1000 \mathrm{~m},-400 \mathrm{~m}]$, most stratified events are indicated with bold circles.

as shown on time depth section of the density (see Fig. 12, especially wave crests between $[-1000 \mathrm{~m},-400 \mathrm{~m}])$. Furthermore, we checked that double diffusion can be dismissed since the stability condition is verified (Turner, 1973):

$\frac{R a}{R a_{\mathrm{s}}} \approx 0.17<\frac{\operatorname{Pr}+\tau}{1+P r}=0.87$,

where $R a$ and $R a_{\mathrm{s}}$ are the Rayleigh and saline Rayleigh number and $\tau$ the ratio between saline and thermal diffusivities. The mean buoyancy frequency is equal to $2.7 \times 10^{-3} \mathrm{rad} / \mathrm{s}$. Over this region the mean density variance is equal to $2.3 \times$ $10^{-4}\left(\mathrm{~kg} / \mathrm{m}^{3}\right)^{2}$ which is much larger than the experimental error of about $5 \times 10^{-6}$.

We first investigated the vertical density structure to search for intermittency. Fig. 13 shows pdf of density differences computed for different vertical separations. Similarly to the numerical simulations, there is an increasing asymmetry as the vertical separation decreases while the width of the distribution increases. This asymmetry is correlated with the occurrence of overturning events $(\Delta \rho>0)$ and is observed for vertical separations smaller than $32 \mathrm{~m}$. Comparison with the pdf of temperature and salinity differences confirms that this strong skewness is associated with the occurrence of static instabilities in a stratified medium. Indeed, the pdfs of temperature differences are symmetric even at small vertical scales (Figs. 14A, C and E), resulting from the fact that the mean vertical gradient is very small $\left(1.14 \times 10^{-4}{ }^{\circ} \mathrm{C} / \mathrm{m}\right)$. In contrast, the pdfs of salinity differences that are strongly skewed at small scales correspond to a stable mean salinity profile.

We next identified the strongly stratified regions seen on the pdf at small scales (asymmetric negative values in Figs. 13A-C). To this aim we selected pairs of locations for which the density difference for $\Delta z=4 \mathrm{~m}$ is larger (in absolute value) than 7 times the mean square root. Nine events were thus obtained that are represented with bold circles in Fig. 12 on the potential density 

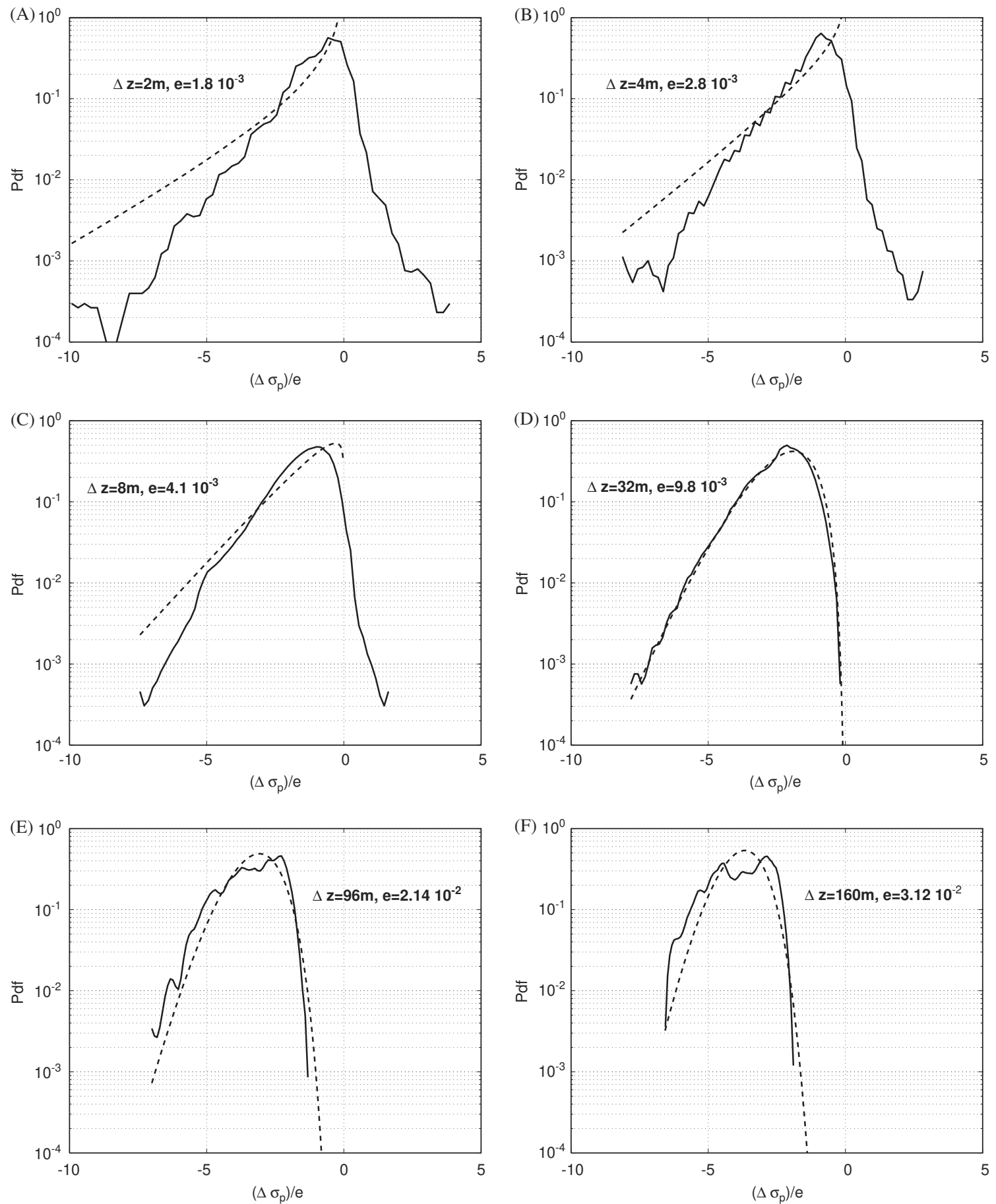

Fig. 13. Probability density functions of density differences for successive vertical separations $\Delta z$ for depth within $[-1000 \mathrm{~m},-400 \mathrm{~m}]$, normalized at each scale by the standard deviation $e$ (given in $\mathrm{kg} / \mathrm{m}^{3}$ ). Prediction of the Hayes et al. model with parameter $\mu=$ $0.16 \mathrm{~m}^{-1}$ (see text) is represented with a dashed line. (A) $\Delta z=2 \mathrm{~m}$, (B) $\Delta z=4 \mathrm{~m}$, (C) $\Delta z=8 \mathrm{~m}$, (D) $\Delta z=32 \mathrm{~m},(\mathrm{E}) \Delta z=96 \mathrm{~m},(\mathrm{~F})$ $\Delta z=160 \mathrm{~m}$. 

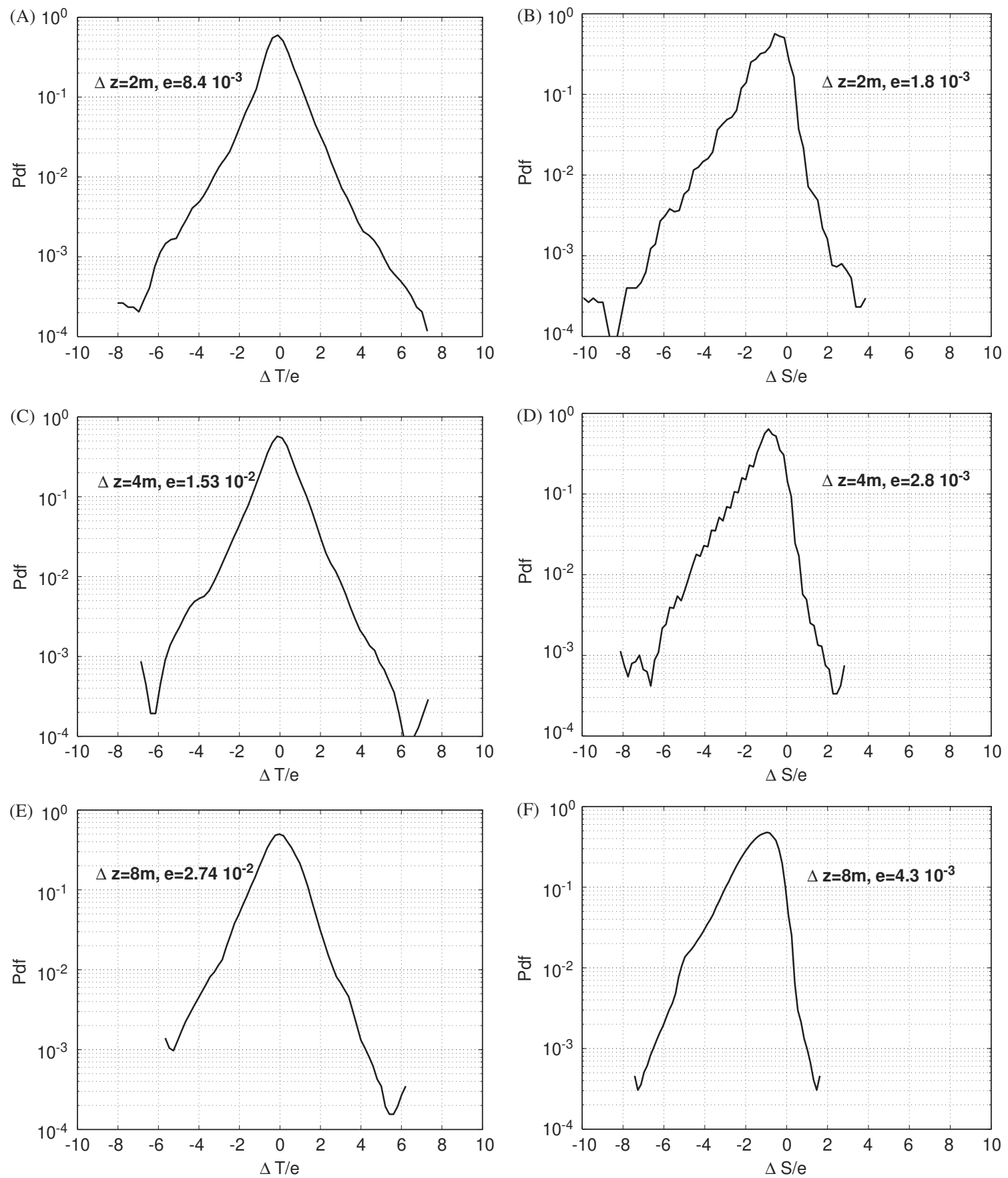

Fig. 14. Probability density functions of temperature and salinity differences for successive vertical separations $\Delta z$ for depth within $[-1000 \mathrm{~m},-400 \mathrm{~m}]$, normalized at each scale by the standard deviation $e$ (given in ${ }^{\circ} \mathrm{C}$ and in ppt, respectively, for $T$ and $S$ ). (A) pdf( $\Delta T$ ) at $\Delta z=2 \mathrm{~m},(\mathrm{~B}) \operatorname{pdf}(\Delta S)$ at $\Delta z=2 \mathrm{~m},(\mathrm{C}) \operatorname{pdf}(\Delta T)$ at $\Delta z=4 \mathrm{~m},(\mathrm{D}) \operatorname{pdf}(\Delta S)$ at $\Delta z=4 \mathrm{~m},(\mathrm{E}) \operatorname{pdf}(\Delta T)$ at $\Delta z=8 \mathrm{~m},(\mathrm{~F}) \operatorname{pdf}(\Delta S)$ at $\Delta z=8 \mathrm{~m}$. 
map. Profiles of the vertical density gradient plotted at the time of occurrence of these events give better evidence of the density steps, three of them are represented in Figs. 15A-C. The four main density steps are indicated by arrows in these figures.

A zoom on the event occurring at $t=13 \mathrm{~h}$ is shown in Fig. 16. A strong density step is observed while overturning events occur on both sides, in a similar way as in the numerical simulations, thus building up the step. Note that lateral thermohaline intrusions do not contribute significantly to this process since a linear relationship between $T$ and $S$ is obtained (Fig. 16B). Zooms on salinity and temperature profiles give evidence of their relative contribution to density variations. The variations of salinity leading to a density overturning play here a major role in building up the density sheet. Indeed, a strong salinity step is observed, that is able to over-compensate the inverse temperature step producing a strong density sheet. Moreover, an inverse temperature sheet is generated by convergent motions produced by breaking events in an unstable background temperature profile.
We have tested the Hayes et al. model against our oceanic data. We proceeded in a similar way as in the numerical simulations. The mean density gradient is inferred from the evolution of mean density differences as a function of vertical separation (Fig. 17A) and the parameter $\mu$ was inferred from the variance of density differences (Fig. 17B). Note that the linear fit was performed in an interval that is consistent with that of the observed skewness of the pdf: $\Delta z \approx[2 \mathrm{~m}, 32 \mathrm{~m}]$. A value of $5.9 \mathrm{~m}$ is obtained for $\mu^{-1}$, which using $\mu^{-1} \approx 5.3 \lambda_{0}$, corresponds to an Ozmidov scale, as previously defined, of $1.1 \mathrm{~m}$, and a total energy dissipation of $\varepsilon=2.4 \times 10^{-8} \mathrm{~W} / \mathrm{kg}$. If we further assume a mixing efficiency of 0.2 (Oakey, 1982) this leads to an estimate of the mean eddy diffusivity $K_{\mathrm{d}}=(\gamma /(\gamma+1)) \lambda_{0}^{2} N=5 \times 10^{-4} \mathrm{~m}^{2} \mathrm{~s}^{-1}$. This fairly high value, about 50 times larger than the estimated background value of $10^{-5}$, agrees within a factor of 2 with the eddy diffusivity inferred from the Thorpe scale following Dillon (1982) and is consistent with the presence of strong internal tides (e.g. Lien and Gregg, 2001).

The shape of the pdfs agrees well with the model within the range $\Delta z \approx[4 \mathrm{~m}, 32 \mathrm{~m}]$, as shown in
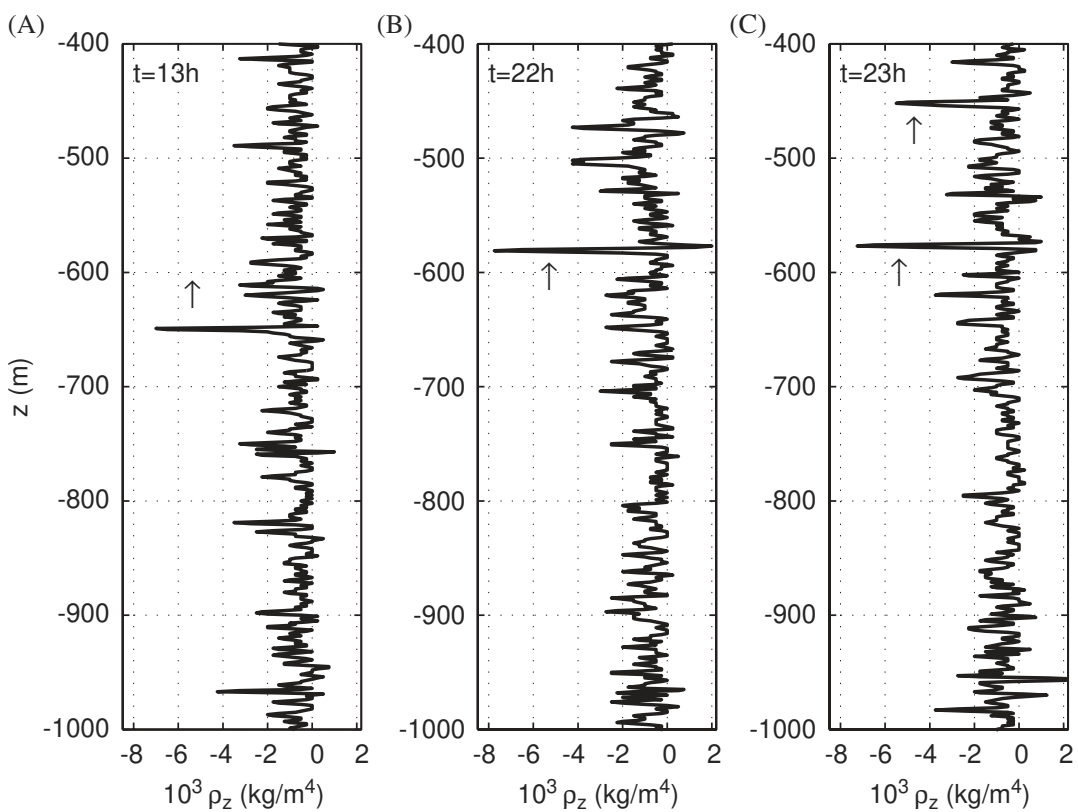

Fig. 15. (A), (B) and (C): Profiles of the vertical density gradient (at $2 \mathrm{~m}$ ) at a given time showing some of the most intense events (indicated by arrows): (A) at time $t=13 \mathrm{~h},(\mathrm{~B})$ at time $t=22 \mathrm{~h},(\mathrm{C})$ at time $t=23 \mathrm{~h}$. 

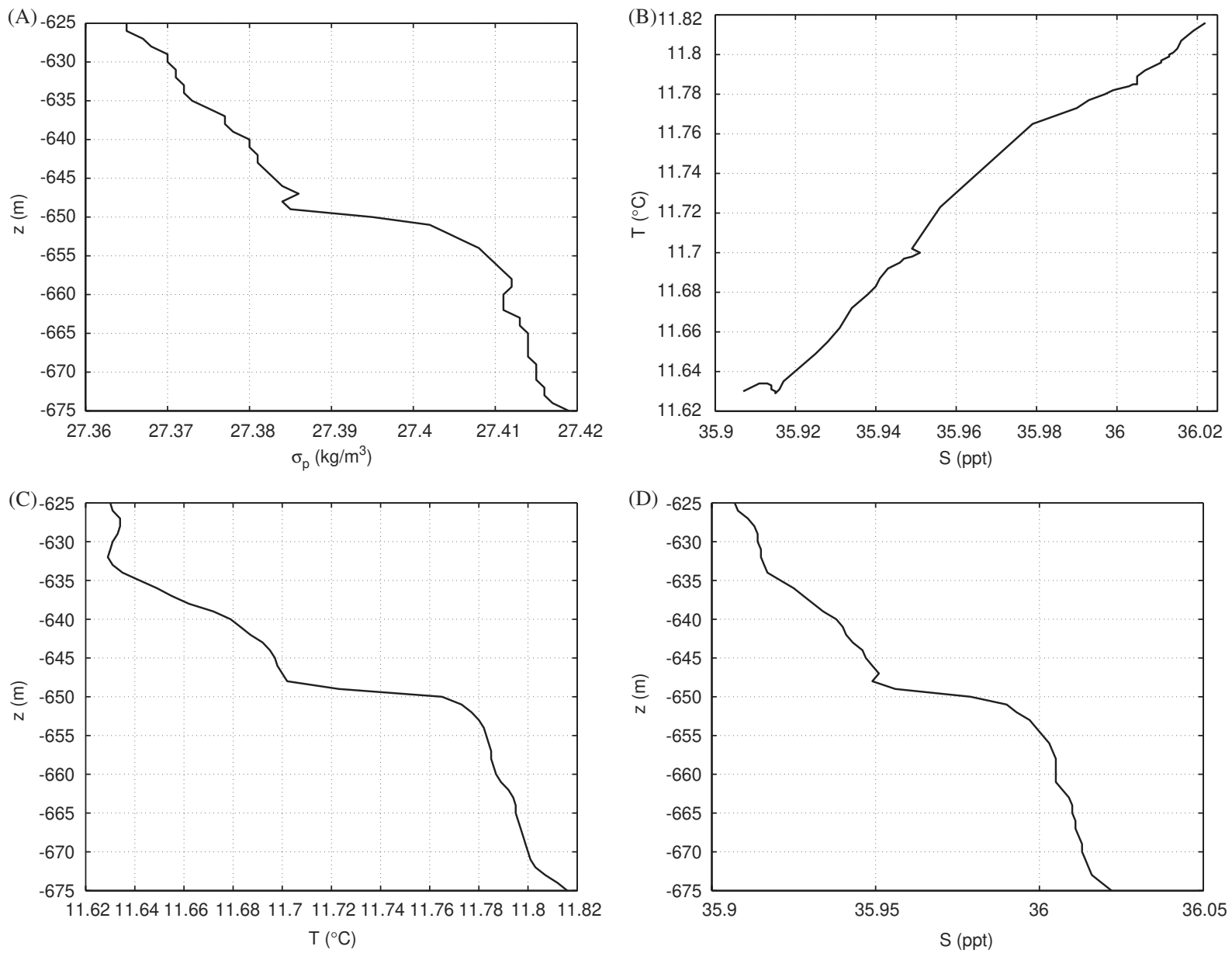

Fig. 16. Zoom over one intense event occurring at $t=13 \mathrm{~h}$ : (A) potential density profile $\sigma_{p}$, (B) vertical potential density gradient profile $\partial_{z} \sigma_{p},(\mathrm{C})$ temperature profile $T$, (D) salinity profile, $S$.

Fig. 13. Also, the percentage of convective regions decreases rapidly as the vertical separation increases. A maximum of $7 \%$ is obtained, that is within the range of our numerical results (see Fig. 4).

\section{Conclusion}

We analyzed some statistical properties of the turbulence induced by the breaking of internal gravity waves. This dynamics corresponds to the so-called buoyancy subrange and it is characterized by kinetic and available potential energy spectra that follow a $k_{z}^{-3}$ power law. In the ocean such behaviour is obtained in the finescale domain, in between weakly nonlinear waves and threedimensional turbulence, corresponding to vertical scales typically within the range $[1 \mathrm{~m}, 10 \mathrm{~m}]$. We focused on the vertical structure of the density field with the main purpose of providing a link between density intermittency, energy dissipation and mixing processes.

Density gradients are strongly intermittent at small scales. The main feature is the strong skewness of the histograms toward stable stratification that becomes evident as soon as overturning events occur. We have characterized these rare and intense events. These take the form of strongly stratified 

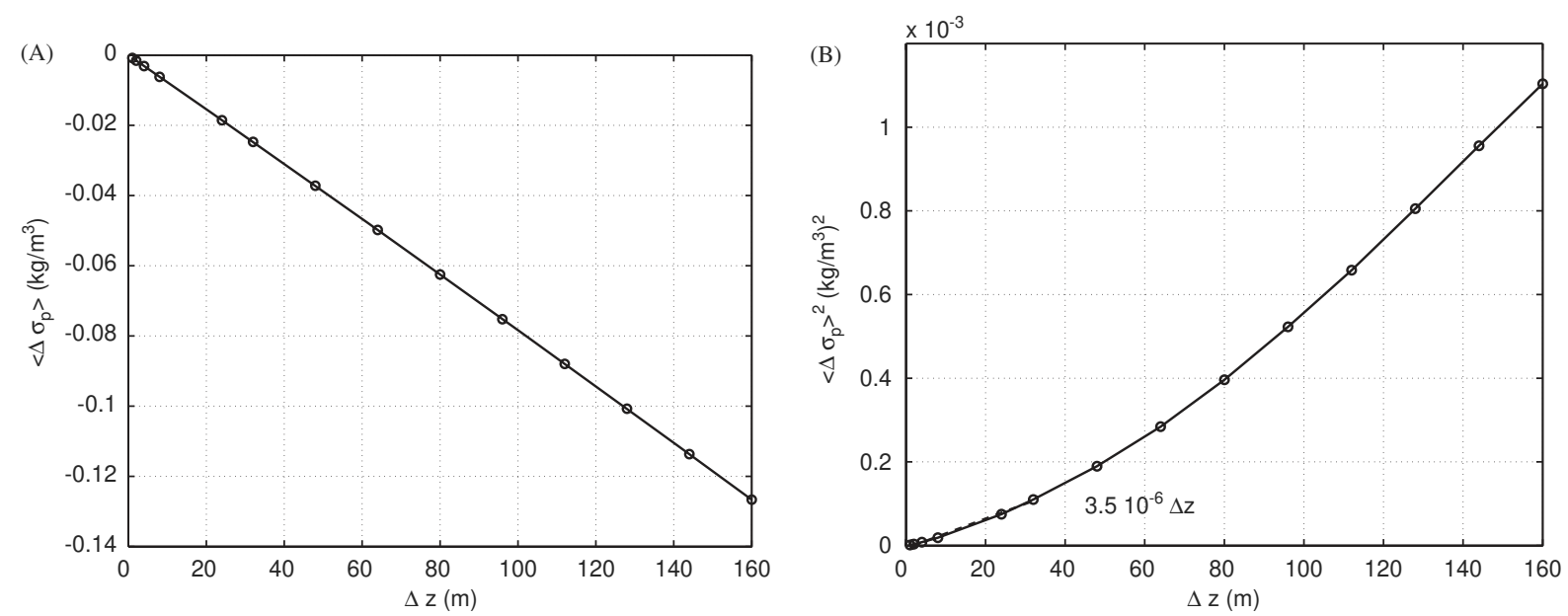

Fig. 17. (A) Mean of $\Delta \sigma_{p}$ as a function of the vertical separation, $\Delta z$; the slope $\beta$ is equal to $7.84 \times 10^{-4}$, (B) Variance of $\Delta \sigma_{p}$ as a function of vertical separation $\Delta z$, the slope $p$ within $[2 \mathrm{~m}, 32 \mathrm{~m}]$ is equal to $3.5 \times 10^{-6}$ and $\mu^{-1}$ is inferred from this linear fit $\mu^{-1}=p / \beta^{2}=5.9 \mathrm{~m}$.

sheets of thin vertical thickness close to the Ozmidov scale and horizontal scale related to that of the large scale breaking wave. These structures are generated through strong convergent motions induced by surrounding overturning events. Indications of these structures were obtained in the atmosphere (e.g. Dalaudier et al., 1994; Alisse and Sidi, 2000) from vertical profiles of temperature, where large stable temperature steps are observed (see Fig. 2 from Alisse and Sidi, 2000). However, because of advection by the mean wind, balloons could not track these sheets. In the ocean the same events seem to be observed on temperature crosssections obtained by Shen and Mied (1986) in the seasonal thermocline of the Sargasso Sea.

We then checked the ability of the sheet and layer model or gamma pdf model (e.g. Hayes et al., 1975; Pinkel and Anderson, 1992) to reproduce the shape of the histograms of vertical density gradient at small vertical scales (typically within $\left\lceil\lambda_{0}, 30 \lambda_{0}\right\rceil$ ). This model, which assumes that the step-like structure is the result of mixing processes, depends on one main parameter, the mean number of sheets (or steps) per unit length, $\mu$. This parameter $\mu$ or alternatively the sheet spacing $\mu^{-1}$ is inferred from the variance and third order moment of density differences over vertical scales within the domain of strong skewness of the pdfs.
The main result of this paper is that the sheet spacing $\mu^{-1}$, varies linearly with the Ozmidov length scale defined with the total energy dissipation rate. Thus this provides a link between density statistics at finescale and energy dissipation. As a consequence, the mean eddy diffusivity that characterizes breaking events varies linearly with a dimensionless parameter defined with $\mu^{-1}$ : $\mu^{-2} N / v$. These findings motivated a preliminary comparison with oceanic data.

The analysis was next being applied to oceanic data from a region of strong internal tides west of the Iberian peninsula. We focused on a dynamically consistent region namely within the interior for depth between -1000 and $-400 \mathrm{~m}$. There, the mean vertical salinity and potential density profiles are stable and linear while the mean vertical temperature gradient is small and unstable in some regions. Thus, density differences are mainly due to salinity variations. Unsurprisingly histograms of density and salinity differences present an increasing skewness at small vertical scales while pdfs of temperature differences are symmetric even at small vertical scales. This underlines that a crucial ingredient to this skewness is the presence of a stable mean vertical gradient. Application of the sheet and layer model to these data gave fairly good predictions for vertical scales within 


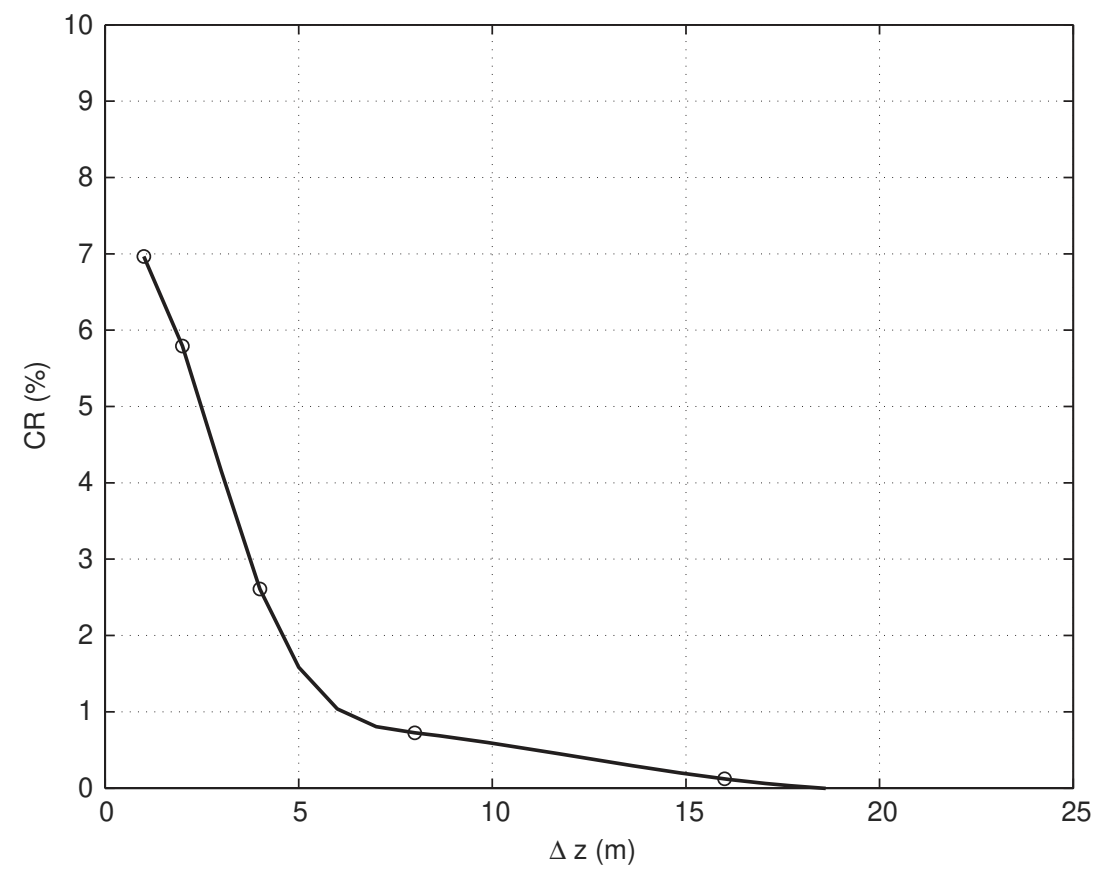

Fig. 18. Percentage of convective regions as a function of the vertical separation $\Delta z$.

[4 m, 32 m]. Finally we applied the relationship between $\mu^{-1}$ and the Ozmidov scale $\lambda_{0}$ obtained from the numerical simulations. This gave estimates of energy dissipation and eddy diffusivity consistent with common values obtained in regions of strong internal tides. In contrast if we apply our numerical results to the Hayes et al. results $\left(\mu^{-1}=0.62 \mathrm{~m}\right)$, an Ozmidov length scale of $12 \mathrm{~cm}$ and an eddy diffusivity of $1.5 \times 10^{-5} \mathrm{~m}^{2} / \mathrm{s}^{-1}$ are obtained. These values are relevant for an open ocean site remote from topographic features.

In future work we plan to further check this indirect estimate of mixing based on density statistics at finescale on a large set of in situ oceanic data and compare this estimate with direct measurements of mixing as inferred from microstructure measurements (Fig. 18).

\section{Acknowledgements}

The authors wish to thank F. Dalaudier for helpful discussions and N. Daniault for her comments regarding the analysis of the oceanic data. Numerical simulations were performed on the NEC SX-5 and the Cray T3E of the Institut du développement et des Ressources en Informatique Scientifique (IDRIS) under contracts 020580 and 980580. PBA acknowledges financial support from ACI CNRS "Jeunes chercheurs" No. 0693 and PATOM/CNRS.

\section{References}

Alisse, J.R., Sidi, C., 2000. Experimental probability density functions of small-scale fluctuations in the stably stratified atmosphere. Journal of Fluid Mechanics 402, 137-162.

Baker, M.A., Gibson, C.H., 1987. Sampling turbulence in the stratified ocean: statistical consequences of strong intermittency. Journal of Physical Oceanography 17, 1817-1836.

Barry, M.E., Ivey, G.N., Winters, K.B., Imberger, J., 2001. Measurements of diapycnal diffusivities in stratified fluids. Journal of Fluid Mechanics 442, 267-291.

Bénielli, D., Sommeria, J., 1998. Excitation and breaking of internal gravity waves by parametric instability. Journal of Fluid Mechanics 374, 117-144.

Bouruet-Aubertot, P., Sommeria, J., Staquet, C., 1995. Breaking of standing internal gravity waves through twodimensional instabilities. Journal of Fluid Mechanics 285, 265-301. 
Bouruet-Aubertot, P., Sommeria, J., Staquet, C., 1996. Stratified turbulence produced by internal wave breaking. Dynamics of Atmospheres and Oceans 23, 371-378.

Bouruet-Aubertot, P., Koudella, C.R., Staquet, C., Winters, K.B., 2001. Particle dispersion and mixing by breaking internal gravity waves. Dynamics of Atmospheres and Oceans 33, 95-134.

Bouruet-Aubertot, P., Sommeria, J., Koudella, C.R., 2004. Intermittency in stratified turbulence produced by breaking internal gravity waves. Journal of Fluid Mechanics, submitted for publication.

Canuto, C., Hussaini, M.Y., Quateroni, A., Zang, T.A., 1988. Spectral Methods in Fluid Dynamics, Springer Series in Computational Physics. Springer, Berlin.

Carnevale, G.F., Briscolini, M., Orlandi, P., 2001. Buoyancy to inertial range transition in forced stratified turbulence. Journal of Fluid Mechanics 427, 205-239.

Dalaudier, F., Sidi, C., Crochet, M., Vernin, J., 1994. Direct evidence of "sheets" in the atmospheric temperature field. Journal of Atmospheric Sciences 51, 237-248.

Desaubies, Y., Gregg, M.C., 1981. Reversible and irrreversible finestructure. Journal of Physical Oceanography 11, 541-556.

Dillon, T.M., 1982. Vertical overturns: a comparison of Thorpe and Ozmidov length scales. Journal of Geophysical Research 87, 9601-9613.

Gargett, A.E., Hendricks, P.J., Stanford, T.B., Osborn, T.R., Williams III, A.J., 1981. A composite spectrum of vertical shear in the ocean. Journal of Physical Oceanography 11, $1258-1271$.

Gregg, M.C., D’Asaro, E.A., Shay, T.J., Larson, N., 1986. Observations of persistent mixing and near-inertial internal waves. Journal of Physical Oceanography 16, 856-885.

Gregg, M.C., Seim, H.E., Percival, D.B., 1993. Statistics of shear and turbulent dissipation profiles in random internal wave fields. Journal of Physical Oceanography 23, 1777-1799.

Hayes, S.P., Joyce, T.M., Millard, J.R., 1975. Measurements of vertical fine structure in the Sargasso Sea. Journal of Geophysical Research 80 (3), 314-319.

Klostermeyer, J., 1991. Two and three-dimensional parametric instabilities in finite amplitude internal gravity waves. Geophysical Astrophysical Fluid Dynamics 61 (1-4), 1-25.
Koudella, C.R., 1999. Ondes internes de gravité: instabilités, déferlement et vorticité potentielle. Thèse de doctorat, Ecole Normale Supérieure de Lyon.

Le Cann, B., Billant, A., Branellec, P., 1998. Campagne ARCANE 96, recueil de données: vol. 1: CTD-O2, Rapport interne LPO 98-01.

Lien, R.C., Gregg, M.C., 2001. Observations of turbulence in a tidal beam and across a coastal ridge. Journal of Geophysical Research 106 (C3), 4575-4591.

Lombard, P.N., Riley, J.J., 1996. Instability and breakdown of internal gravity waves: I. linear stability analysis. Physics of Fluids 8 (12), 3271-3287.

Oakey, N.S., 1982. Determination of the rate of dissipation of turbulent energy from simultaneous temperature and velocity shear microstructure measurements. Journal of Physical Oceanography 12, 256-271.

Olbers, D.J., 1976. Non-linear energy transfers and the energy balance of the internal wave field in the deep ocean. Journal of Fluid Mechanics 74, 375-399.

Osborn, T.R., 1980. Estimates of the local rate of vertical diffusion from dissipation measurements. Journal of Physical Oceanography 10, 83-89.

Osborn, T.R., Cox, C.S., 1972. Oceanic finestructure. Geophysical Fluid Dynamics 3, 321-345.

Pinkel, R., Anderson, S., 1992. Toward a statistical description of finescale strain in the thermocline. Journal of Physical Oceanography 22 (7), 773-795.

Pinkel, R., Anderson, S., 1997a. Shear, strain and Richardson number variations in the thermocline. Part I: statistical description. Journal of Physical Oceanography 27 (2), 264-281.

Pinkel, R., Anderson, S., 1997b. Shear, strain and Richardson number variations in the thermocline. Part II: modelling mixing. Journal of Physical Oceanography 27 (2), 282-290.

Turner, J.S., 1973. Buoyancy Effects in Fluids. Cambridge University Press, Cambridge.

Winters, K.B., D'Asaro, E.A., 1996. Diascalar flux and the rate of fluid mixing. Journal of Fluid Mechanics 317, 179-193.

Yamazaki, H., Lueck, R., 1990. Why oceanic dissipation rates are not lognormal. Journal of Physical Oceanography 20, 1907-1918. 